\title{
The global mass-to-light ratio of SLACS lenses
}

\author{
V. F. Cardone $e^{1,2,3}$, C. Tortora ${ }^{1,2,4}$, R. Molinaro ${ }^{5}$, and V. Salzano ${ }^{1,6}$ \\ 1 Dipartimento di Scienze Fisiche, Università di Napoli Federico II, Complesso Universitario Monte S. Angelo - Edificio 6 , \\ via Cinthia, 80126 Napoli, Italy \\ e-mail: winnyenodrac@gmail.com \\ 2 Osservatorio Astrofisico di Catania, via Santa Sofia 78, 95123 Catania, Italy \\ 3 Dipartimento di Fisica Generale "A. Avogadro", Università di Torino and Istituto Nazionale di Fisica Nucleare, Sezione di Torino, \\ via Pietro Giuria 1, 10125 Torino, Italy \\ 4 Osservatorio Astronomico di Capodimonte, Salita Moiariello 16, 80131 Napoli, Italy \\ 5 Dipartimento di Fisica, Politecnico di Torino, Corso Duca degli Abruzzi 24, 10129 Torino, Italy \\ ${ }^{6}$ Istituto Nazionale di Fisica Nucleare, sezione di Napoli, Complesso Universitario di Monte S. Angelo, Edificio 6, via Cinthia, \\ 80126 Napoli, Italy
}

Received 5 October 2008 / Accepted 27 May 2009

\section{ABSTRACT}

\begin{abstract}
Aims. The dark matter content of early-type galaxies (ETGs) is a hotly debated topic with contrasting results arguing in favour of or against the presence of significant dark mass within the effective radius and the change with luminosity and mass. To address this question, we investigate here the global mass-to-light ratio $\Upsilon(r)=M(r) / L(r)$ of a sample of 21 lenses observed within the Sloan Lens ACS (SLACS) survey.

Methods. We follow the usual approach of the galaxy as a two component systems, but we use a phenomenological ansatz for $\Upsilon(r)$, proposed by some of us, that is able to smoothly interpolate between constant $M / L$ models and a wide class of dark matter haloes. The resulting galaxy model is then fitted to the data on the Einstein radius and aperture velocity dispersion.

Results. Our phenomenological model turns out to agree with the data suggesting the presence of massive dark matter haloes to explain the lensing and dynamics properties of the SLACS lenses. According to the values of the dark matter mass fraction, we argue that the halo may play a significant role in the inner regions probed by the data, but such a conclusion strongly depends on the adopted initial mass function of the stellar population. Finally, we find that the dark matter mass fraction within $R_{\text {eff }}$ scales with both the total luminosity and stellar mass in such a way that more luminous (and hence more massive) galaxies have a greater dark matter content.
\end{abstract}

Key words. galaxies: kinematics and dynamics - galaxies: fundamental parameters - galaxies: elliptical and lenticular, $\mathrm{Cd}-$ gravitational lensing - cosmology: dark matter

\section{Introduction}

Early-type galaxies (hereafter ETGs) represent the most massive and brightest stellar systems in the universe. Notwithstanding the regularity of their photometric properties and the existence of remarkable scaling relations, detailed analysis of their structure has been plagued by both observational and theoretical shortcomings. Such a frustrating situation mainly stems from the lack of a reliable mass tracer able to probe the mass profile outside the effective radius. Although the use of planetarey nebulae have recently improved the observational situation allowing the gravitational potential in the outer regions (Napolitano et al. 2001, 2002; Romanowsky et al. 2003) to be probed, the well known mass-anisotropy degeneracy still represent s a theoretical shortcoming preventing a unique reconstruction of the mass profile.

Numerical simulations of galaxy formation are usually invoked as a guide towards understanding the structure of the dark matter (DM) haloes. Unfortunately, while there is a general consensus on the mass density profile $\rho_{\mathrm{DM}}(r)$ being correctly described by a double-power law function with an outer slope $s=\mathrm{d} \ln \rho / \mathrm{d} \ln r=-3$, there is still an open debate concerning the inner scaling of $\rho_{\mathrm{DM}}(r)$. The pioneering result $\rho_{\mathrm{DM}} \sim r^{-1}$ of Navarro et al. $(1996,1997)$ has been questioned by other simulations providing either a steeper cusp (e.g., Moore et al. 1998; Ghigna et al. 2000; Jing \& Suto 2000; Fukushige \& Makino 2001) or a shallow profile (e.g., Power et al. 2003; Navarro et al. 2004). Needless to say, galaxies are two component systems with the luminous matter playing a not negligible role in the inner regions which are indeed the most well probed by the data. While there is general agreement on the existence and dominance of DM in the outer galaxy regions, where stars are practically absent (see, e.g., van den Bosch et al. 2003a,b, 2007), paradoxically the DM content in the inner regions is more difficult to interpret notwithstanding the availability of a higher number of possible tracers. As pointed out by Mamon \& Lokas (2005a), the observational data claim for a dominant stellar component at a radius $\lesssim 1 R_{\text {eff }}$. However, the uncertainties on which is the stellar initial mass function (IMF), with the Salpeter (1995) and Chabrier (2001) as leading but not unique candidates, makes it quite difficult to assess the general validity of such a result. On large scales, the DM content has been found to be a strong function of both luminosity and mass (Benson et al. 2000; Marinoni \& Hudson 2002; van den Bosch et al. 2007) with a different behaviour between faint and bright systems. Looking for a similar result for the DM content within $\sim 1 R_{\text {eff }}$ is quite controversial. On one hand, 
some authors (Gerhard et al. 2001; Borriello et al. 2003) argue for no dependence. In contrast, other works (e.g., Napolitano et al. 2005; Cappellari et al. 2006; Tortora et al. 2009b) do find that brighter galaxies have a larger DM content, while a flattening and a possible inversion of this trend for lower mass systems, similar to the one observed for late-type galaxies (Persic et al. 1993), is still under analysis (Napolitano et al. 2005; Tortora et al. 2009b) with no conclusive result yet obtained.

Given the controversial situation for local galaxies, it is not surprising that the analysis of high redshift galaxies is still more difficult considering that only partial information are usually recovered. For instance, rather than a full velocity dispersion profile, typically one is able to measure only a single value describing the global velocity dispersion of the galaxy. This lack of radial data extent obviously limits the analysis representing a strong obstacle to any attempts to constrain the galaxy mass profile. Fortunately, a different mass tracer may be available for a subset of ETGs at intermediate redshift. Actually, gravitational lensing gives a strong constraint on the projected mass within the so called Einstein radius (Schneider et al. 1992; Petters et al. 2001). It is worth stressing that such an observational quantity is fully model independent although a galaxy model has to be assumed to extrapolate the results to other radii. Combining strong lensing with the information from galaxy dynamics makes it possible to not only increase the number of constraints, but also to partially break the mass - anisotropy degeneracy (Treu \& Koopmans 2002a; Koopmans \& Treu 2003a; Bolton et al. 2008) thus representing a powerful tool to probe mass profiles for lens systems.

It is worth noting that most of the analyses in literature heavily rely on the choice of an analytical expression for the density profile of the DM halo. Needless to say, this makes the results model dependent with the risk of biasing them in a difficult to quantify way. Here, in order to escape this problem, we follow the approach presented by some of us in Tortora et al. (2007) where a phenomenological ansatz for the global $M / L$ ratio $\Upsilon(r)=M(r) / L(r)$ has been proposed. Such a model is able to smoothly interpolated between constant $M / L$ models (where light traces mass) and the different DM haloes profiles thus providing a unifying description of a large class of total mass profiles. Fitting the model to the lensing and dynamics observables makes it possible to constrain its characterizing parameters thus giving constraints on the DM mass content and its dependence on the luminosity or mass of ETGs at intermediate redshift. As a further step on with respect to our original paper, we here improve the analysis by first investigating which region of the parameter space is better suited to mimic the most used dark halo models. We then fit our model to the observed data on lensing and dynamics in order to individuate, within the plethora of possible trends admitted by our parametric choice for $M / L$ ratio, the best ones able to match observations. Such an analysis then makes it also possible to infer constraints on the DM mass fraction and investigate whether any dependence on luminosity and stellar mass can be detected in these intermediate redshift systems.

The paper is organized as follows. In Sect. 2, we introduce our parametrization of the global $M / L$ ratio and discuss the main properties of the effective galaxy model coming out from the combination of our ansatz for $\Upsilon(r)$ and an approximate analytical deprojection of the Sersic surface brightness profile. The lensing properties on the model are discussed in Sect. 3 where we work out expressions for the deflection and Einstein angle. The statistical methodology used to contrast the model against the observations and the dataset are presented in Sect. 5, while a detailed discussion of the results is the subject of Sect. 6. We finally summarize and conclude in Sect. 7. Along the paper, where needed, distances are converted from angular to physical units adopting a flat $\Lambda \mathrm{CDM}$ cosmological model with parameters $\left(\Omega_{\mathrm{M}}, \Omega_{\Lambda}, h\right)=(0.3,0.7,0.7)$ (Spergel et al. 2003).

\section{The model}

The lack of a reliable mass tracer in the outer regions of ETGs makes the problem of DM content in these systems still open and hotly debated. In the usual approach, a dark halo model, described by a given density profile, is added to the visible component and then used to fit a given dataset (e.g., the velocity dispersion profile or the X-ray emission). Needless to say, such an approach is strongly model dependent and plagued by parameter degeneracies so that the constraints on, e.g., the DM mass fraction within the effective radius $R_{\text {eff }}$ are possibly affected by a theoretical prejudice. In order to overcome such a problem, some of us have recently advocated the use of a different strategy. Rather than separately modeling the two galaxy components, we have proposed to directly constrain its total (luminous plus dark) cumulative mass profile $M(r)$. Under the assumption of spherical symmetry, it is possible to write it as:

$$
M(r)=M_{\star}(r)+M_{\mathrm{DM}}(r)=\Upsilon(r) L(r)
$$

where $M_{\star}\left(M_{\mathrm{DM}}\right)$ is the mass distribution of the luminous (dark) component, $L(r)$ the integrated luminosity profile, and $\Upsilon(r)$ is the global $M / L$ ratio. Since $L(R)$ may be reconstructed (at least, in principle) by deprojecting the observed surface brightness, the only unknown ingredient is just $\Upsilon(r)$. On the one hand, should the DM content be negligible, which is a borderline case of a constant $M / L$ model, one should expect $\Upsilon(r)$ to remain approximately constant to the stellar $M / L$ value. In contrast, $\Upsilon(r)$ will be an increasing function of $r$ if the dark halo plays a significant role since the total mass $M(r)$ should overcome the stellar one in the outer regions. A unified description of these two opposite trends is provided by the following phenomenological ansatz (Tortora et al. 2007):

$$
\begin{aligned}
\Upsilon(r) & =\Upsilon_{0}\left(\frac{r}{r_{0}}\right)^{\alpha}\left(1+\frac{r}{r_{0}}\right)^{\beta} \\
& =\Upsilon_{0}\left(\frac{\eta}{\eta_{0}}\right)^{\alpha}\left(1+\frac{\eta}{\eta_{0}}\right)^{\beta} \\
& =\Upsilon_{\mathrm{eff}}\left(\frac{\eta+\eta_{0}}{1+\eta_{0}}\right)^{\beta} \eta^{\alpha}
\end{aligned}
$$

with $\eta=r / R_{\mathrm{eff}}, \eta_{0}=r_{0} / R_{\mathrm{eff}}$ and

$\Upsilon_{\mathrm{eff}}=\frac{\Upsilon_{0}\left(1+\eta_{0}\right)^{\beta}}{\eta_{0}^{\alpha+\beta}}$

While $\left(\Upsilon_{0}, r_{0}\right)$ are scaling quantities, the two parameters $(\alpha, \beta)$ plays a more significant role setting the asymptotic slopes of the global $M / L$ ratio. Indeed, it is:

$\Upsilon(r) \propto \begin{cases}\Upsilon_{0}\left(r / r_{0}\right)^{\alpha} & r \ll r_{0} \\ \Upsilon_{0}\left(r / r_{0}\right)^{\alpha+\beta} & r \gg r_{0} .\end{cases}$

Equation (3) clearly shows that, rather than $\beta$, it is $\alpha+\beta$ the important quantity so that, in the following, we will parametrize 
the model by the parameters $\left(\alpha, \alpha+\beta, \eta_{0}, \Upsilon_{\mathrm{eff}}\right)$. It is instructive to look at the following limits:

$\lim _{\eta \rightarrow 0} \Upsilon(\eta)=\left\{\begin{array}{cc}0 & \alpha>0, \alpha+\beta>0 \\ \infty & \alpha<0, \alpha+\beta>0 \\ 0 & \alpha>0, \alpha+\beta<0 \\ \infty & \alpha<0, \alpha+\beta<0,\end{array}\right.$

while for $\alpha=0$, we get:

$\lim _{\eta \rightarrow 0} \Upsilon(\eta)=\Upsilon_{0}$

According to Eqs. (4) and (5), the global $M / L$ diverges if $\alpha$ is negative. Considering its definition and that both $M(r)$ and $L(r)$ identically vanish in the origin, we shall conclude that, for this class of models, $M(r)$ goes to zero faster than $L(r)$ which is not expected. Indeed, should visible mass dominate in the inner regions, we expect $M(r) \simeq M_{\star}(r)=\Upsilon_{*} L(r)$, with $\Upsilon_{*}$ the stellar $M / L$ ratio, so that $M(r)$ and $L(r)$ decreases with the same rate. In the opposite case, i.e. DM is more concentrated than the light, it is possible to have $M(r)$ decreasing slower than light so that we cannot discard models with negative $\alpha$. On the other hand, when $\alpha>0$, we get a null global $M / L$ ratio in the origin which is an unphysical result since $\Upsilon(r)$ can never become smaller than the stellar $M / L$. For this same reason, the case $\alpha=0$ should be accepted provided that $\Upsilon_{0} \geq \Upsilon_{\star}$ hold.

One could then argue that the case $\alpha>0$ should be discarded. It is worth stressing, however, that our ansatz in Eq. (1) is purely phenomenological. We have looked for a functional shape that makes it possible to fit as well as possible different realistic models suitably adjusting the characteristic parameters. As for all fitting expressions, we do not expect that the fitted approximation recovers the underlying dark halo model from zero to infinity, but only over a limited (although large) radial range. As we will see later, this is what indeed happens with our parametrization of $\Upsilon(r)$ notwithstanding the very inner profile shape. Motivated by these considerations, we will therefore consider both $\alpha \geq 0$ and $\alpha<0$ models.

As an attempt to constrain $(\alpha+\beta)$, we can consider the following limits:

$\lim _{\eta \rightarrow \infty} \Upsilon(\eta)= \begin{cases}\infty & \alpha \geq 0, \alpha+\beta>0 \\ \infty & \alpha<0, \alpha+\beta>0 \\ 0 & \alpha \geq 0, \alpha+\beta<0 \\ 0 & \alpha<0, \alpha+\beta<0,\end{cases}$

which show that models with $\alpha+\beta<0$ are clearly unphysical. Indeed, in this case, we easily get $M(r \rightarrow \infty)=0$, that is to say the total mass of the system is null. Actually, as we will see later, it is not possible to reproduce the $\Upsilon(r)$ trend of any DM model with our ansatz (1) when $\alpha+\beta<0$ so that we will henceforth only consider models with nonnegative values of the $\alpha+\beta$ parameter (Tortora et al. 2007). In this case, the total mass is divergent, but this is a common feature for many dark halo models (e.g., the isothermal sphere and the NFW profile). In order to avoid this problem, one may truncate the halo at the virial radius.

Further hints on the role of the different parameters at work may be obtained considering the logarithmic slope of the global $M / L$ ratio. It is:

$\frac{\mathrm{d} \log \Upsilon(\eta)}{\mathrm{d} \log \eta}=\alpha+\beta \eta\left(\eta+\eta_{0}\right)^{-1}$ so that:

$$
\frac{\mathrm{d} \log \Upsilon(\eta)}{\mathrm{d} \log \eta} \geq 0 \Longleftrightarrow \eta \geq \eta_{\mathrm{c}}=-[\alpha /(\alpha+\beta)] \eta_{0} .
$$

Since we have decided to only consider models with $\alpha+\beta>0$, we therefore conclude that the global $M / L$ ratio is everywhere increasing if $\alpha>0$, while the logarithmic slope becomes positive only after the threshold radius $\eta_{\mathrm{c}}$ when $\alpha$ is negative. As we will see, both these trends are possible for realistic DM models so that we will not set any constraint on $\eta_{\mathrm{c}}$. It is worth noting that, in a narrow range around $\eta_{\mathrm{c}}$, the logarithmic slope is approximately null so that $\Upsilon(\eta)$ is constant as in the mass-traceslight models. Should the data at hand probe just this radial range, one can reasonably fit them with a constant $M / L$ ratio model.

\subsection{The luminosity density}

The phenomenological ansatz for $\Upsilon(r)$ is just one side of the coin, the other one being the choice of the luminosity profile. In principle, this quantity may be directly reconstructed from the data by first deconvolving the observed surface brightness with the PSF of the images and then deprojecting it under an assumption for the intrinsic flattening. Needless to say, such an idealized procedure is strongly affected by noise and will call for a case by - case study. Fortunately, ETGs are quite regular in their photometric properties. Indeed, as well known (Caon et al. 1993; Graham \& Colless 1997; Prugniel \& Simien 1997), their surface brightness is well described by the Sersic profile (Sersic 1968):

$I(R)=I_{\mathrm{e}} \exp \left\{-b_{n}\left[\left(\frac{R}{R_{\mathrm{eff}}}\right)^{1 / n}-1\right]\right\}$

with $R$ the cylindrical radius ${ }^{1}$ on the plane of the sky and $I_{\mathrm{e}}$ the luminosity intensity at the effective radius $R_{\text {eff }}$. The constant $b_{n}$ is determined by the condition that the luminosity within $R_{\text {eff }}$ is half the total luminosity, i.e. $b_{n}$ is found by solving:

$\Gamma\left(2 n, b_{n}\right)=\Gamma(2 n) / 2$

where $\Gamma(a, z)$ is the incomplete $\Gamma$ function. Although Eq. (9) is straightforward to solve numerically, a very good analytical approximation is given by (Ciotti \& Bertin 1999):

$b_{n}=2 n-\frac{1}{3}-\frac{0.009876}{n}$

The deprojection of the intensity profile in Eq. (8) is straightforward under the hypothesis of spherical symmetry, but, unfortunately, the result turns out to be a somewhat involved combinations of the unusual Meijer functions (Mazure \& Capelato 2002). In order to not deal with these difficult to handle expression, we prefer to use the model proposed by Prugniel \& Simien (1997, hereafter PS97) whose three dimensional luminosity density reads:

$j(r)=j_{0}\left(\frac{r}{R_{\mathrm{eff}}}\right)^{-p_{n}} \exp \left[-b_{n}\left(\frac{r}{R_{\mathrm{eff}}}\right)^{1 / n}\right]$

\footnotetext{
${ }^{1}$ Note that we have implicitly assumed that the intensity $I$ does not depend on the angular coordinates. Actually, the isophotes are not concentric circles, but rather ellipses with variable ellipticities and position angles so that $I=I(R, \varphi)$. However, in order to be consistent with our assumption of spherical symmetry of the three dimensional mass profile, we will neglect such an effect and, following a common practice, circularize the intensity profile considering circular isophothes with radii equal to the geometric mean of the major and minor axes.
} 
with

$j_{0}=\frac{I_{0} b_{n}^{n\left(1-p_{n}\right)}}{2 R_{\mathrm{eff}}} \frac{\Gamma(2 n)}{\Gamma\left[n\left(3-p_{n}\right)\right]}$.

Here, $I_{0}=I(R=0)=I_{\mathrm{e}} \mathrm{e}^{b_{n}}$, while the constant $p_{n}$ is chosen so that the projection of Eq. (10) matches a Sersic profile with the same values of $\left(n, R_{\mathrm{eff}}, I_{\mathrm{e}}\right)$. A useful fitting formula is given as (Márquez et al. 2001):

$p_{n}=1.0-\frac{0.6097}{n}+\frac{0.00563}{n^{2}}$.

Because of the assumed spherical symmetry, the luminosity profile may simply be obtained as:

$L(r)=4 \pi \int_{0}^{r} r^{\prime 2} j\left(r^{\prime}\right) \mathrm{d} r^{\prime}$

which, for the PS model, becomes:

$L(r)=L_{\mathrm{T}} \times \frac{\gamma\left[n\left(3-p_{n}\right), b_{n} \eta^{1 / n}\right)}{\Gamma\left[n\left(3-p_{n}\right)\right]}$

where the total luminosity $L_{\mathrm{T}}$ reads:

$L_{\mathrm{T}}=2 \pi n b_{n}^{-2 n} \mathrm{e}^{b_{n}} \Gamma(2 n) I_{\mathrm{e}} R_{\mathrm{eff}}^{2}$.

Note that the total luminosity is the same as the projected one for the corresponding Sersic profile as can be immediately check computing:

$L_{\mathrm{T}}=2 \pi \int_{0}^{\infty} I(R) R \mathrm{~d} R$.

It is worth remembering the definitions of the different special functions entering Eqs. (9)-(13):

$\Gamma(a)=\int_{0}^{\infty} t^{a-1} \mathrm{e}^{-t} \mathrm{~d} t$,

$\Gamma(a, y)=\int_{y}^{\infty} t^{a-1} \mathrm{e}^{-t} \mathrm{~d} t$

$\gamma(a, y)=\int_{0}^{y} t^{a-1} \mathrm{e}^{-t} \mathrm{~d} t$

so that the following useful relations hold:

$\Gamma(a)=\lim _{y \rightarrow 0} \Gamma(a, y)=\lim _{y \rightarrow \infty} \gamma(a, y)$

$\gamma(a, y)=\Gamma(a)-\Gamma(a, y)$

As a final remark, let us stress that the total mass of the stellar component $M_{\star}^{\mathrm{T}}=\Upsilon_{*} L_{\mathrm{T}}$ may be determined from the measurement of the photometric parameters $\left(n, R_{\mathrm{eff}}, I_{\mathrm{e}}\right)$ provided that an estimate of the stellar $M / L$ ratio $\Upsilon_{*}$ is available (for instance, from the relation between $\Upsilon_{*}$ and the colours or from fitting the galaxy spectrum to stellar population synthesis models).

\subsection{The total mass, density profile and DM fraction}

Combining Eqs. (1) and (12), we trivially get for the total mass profile:

$$
\begin{aligned}
M(r) & =\Upsilon_{0} L_{\mathrm{T}}\left(\frac{\eta}{\eta_{0}}\right)^{\alpha}\left(1+\frac{\eta}{\eta_{0}}\right)^{\beta} \frac{\gamma\left[n\left(3-p_{n}\right), b_{n} \eta^{1 / n}\right]}{\Gamma\left[n\left(3-p_{n}\right)\right]} \\
& =\Upsilon_{\mathrm{eff}} L_{\mathrm{T}} \frac{\eta^{\alpha}\left(\eta+\eta_{0}\right)^{\beta} \gamma\left[n\left(3-p_{n}\right), b_{n} \eta^{1 / n}\right.}{\left(1+\eta_{0}\right)^{\beta} \Gamma\left[n\left(3-p_{n}\right)\right]} .
\end{aligned}
$$

Formally, $M(r)$ diverges if $\alpha+\beta>0$. However, this is not a serious problem since, following a common practice, we will truncate the model at the virial radius $R_{\mathrm{vir}}$ defined as the radius of the sphere within which the mean density is $\Delta_{c}(z) \bar{\rho}_{M}(z)$ with:

$\bar{\rho}_{M}(z)=\Omega_{0 M} \rho_{\text {crit }}(1+z)^{3}=\left(3 H_{0}^{2} / 8 \pi G\right) \Omega_{0 M}(1+z)^{3}$,

and, following Bryan \& Norman (1998), we approximate the virial overdensity as:

$\Delta_{c}(z)=\frac{18 \pi+82 x(z)-39 x^{2}(z)}{\Omega_{0 \mathrm{M}}}$

where $\Omega_{0 \mathrm{M}}$ is the present day matter density parameter and, for the flat $\Lambda \mathrm{CDM}$ model we are considering, it is:

$x(z)=\Omega_{M}(z)-1=\frac{\Omega_{0 \mathrm{M}}(1+z)^{3}}{\sqrt{\Omega_{0 \mathrm{M}}(1+z)^{3}-\left(1-\Omega_{0 \mathrm{M}}\right)}}-1$.

The total mass is then the virial one obtained by setting $\eta=$ $\eta_{\text {vir }}=R_{\text {vir }} / R_{\text {eff }}$ in Eq. (14) with:

$R_{\mathrm{vir}}=\left[\frac{3 M_{\mathrm{vir}}}{4 \pi \Delta_{c} \bar{\rho}_{M}(z)}\right]^{1 / 3}$,

and solving with respect to $M_{\mathrm{vir}}$. This gives:

$M_{\mathrm{vir}}=\Upsilon_{\mathrm{eff}} L_{\mathrm{T}} \times \frac{\eta_{\mathrm{vir}}^{\alpha}\left(\eta_{\mathrm{vir}}+\eta_{0}\right)^{\beta} \gamma\left[n\left(3-p_{n}\right), b_{n} \eta^{1 / n}\right]}{\left(1+\eta_{0}\right)^{\beta} \Gamma\left[n\left(3-p_{n}\right)\right]}$,

so that one can conveniently rewrite the mass profile as:

$$
\begin{aligned}
M(r)= & M_{\mathrm{vir}} \\
& \times \frac{\left(\eta / \eta_{0}\right)^{\alpha}\left(1+\eta / \eta_{0}\right)^{\beta} \gamma\left[n\left(3-p_{n}\right), b_{n} \eta^{1 / n}\right]}{\left(\eta_{\mathrm{vir}} / \eta_{0}\right)^{\alpha}\left(1+\eta_{\mathrm{vir}} / \eta_{0}\right)^{\beta} \gamma\left[n\left(3-p_{n}\right), b_{n} \eta_{\mathrm{vir}}^{1 / n}\right]}
\end{aligned}
$$

which clearly shows that $\left(R_{\mathrm{eff}}, I_{\mathrm{e}}, \Upsilon_{\mathrm{eff}}\right)$ only works as scaling parameters through $M_{\mathrm{vir}}$, while the shape of $M(r)$ is controlled by the Sersic index $n$ and the $M / L$ slopes $(\alpha, \beta)$. Note that the light traces mass case is only achieved by setting both $\alpha=\beta=0$ and $\Upsilon_{\text {eff }}=\Upsilon_{*}$, with $\Upsilon_{*}$ the stellar $M / L$ ratio. Varying these parameters, Eq. (16) provides a wide range of possible mass profiles thus allowing a rather large class of dark halo models to be mimicked. Note also that, in principle, one should also check that $M(r)$ is a monotonically increasing function of $r$. To this end, it is convenient to consider the logarithmic slope of the mass profile reading:

$$
\begin{aligned}
\frac{\mathrm{d} \ln M}{\mathrm{~d} \ln \eta}= & \frac{\left(b_{n} \eta^{1 / n}\right)^{n\left(3-p_{n}\right)} \mathrm{e}^{-b_{n} \eta^{1 / n}}}{n \gamma\left[n\left(3-p_{n}\right), b_{n} \eta^{1 / n}\right]} \\
& +\frac{\left[\alpha\left(\eta+\eta_{0}\right)+\beta \eta\right] \gamma\left[n\left(3-p_{n}\right), b_{n} \eta^{1 / n}\right]}{\eta+\eta_{0}} .
\end{aligned}
$$

It is instructive to first consider the case $n=4$. We get:

$\lim _{\eta \rightarrow 0} \frac{\mathrm{d} \ln M}{\mathrm{~d} \ln \eta}=\alpha, \lim _{\eta \rightarrow \infty} \frac{\mathrm{d} \ln M}{\mathrm{~d} \ln \eta}=\alpha+\beta$, 
so that only models with $\alpha \geq 0$ and $\alpha+\beta \geq 0$ are viable. Leaving $n$ as a free parameter, we find:

$\frac{\mathrm{d} \ln M}{\mathrm{~d} \ln \eta} \geq 0 \Longleftrightarrow \eta \geq \eta_{\mathrm{c}}=-\frac{\alpha \eta_{0}}{\alpha+\beta}$.

Not surprisingly, this is the same constraint we have obtained when considering the logarithmic slope of the global $M / L$ ratio $\Upsilon(r)$ so that we refer the reader to the above discussion. We only stress here that models with $\alpha<0$ have an unphysical mass profile in the inner regions. As such, the behaviour of these models for such low values of $r$ should not be considered as a good approximation of the reality. Indeed, we get:

$\lim _{\eta \rightarrow 0} M(\eta, \alpha<0)= \pm \infty$,

which is clearly an unphysical result. However, one can consider our ansätz for $\Upsilon(r)$ only as a phenomenological approximation working well everywhere but for $\eta \leq \eta_{\mathrm{c}}$. Since this quantity is typically quite small, hereafter, we will consider both negative and positive values of $\alpha$, while only models with $\alpha+\beta>0$ will be explored to avoid dealing with an unphysical vanishing total mass.

Because of the assumed spherical symmetry, the mass density may be straightforwardly derived as:

$\rho(r)=\frac{1}{4 \pi r^{2}} \frac{\mathrm{d} M}{\mathrm{~d} r}=\frac{1}{4 \pi R_{\mathrm{eff}}^{3}} \frac{1}{\eta^{2}} \frac{\mathrm{d} M}{\mathrm{~d} \eta}$.

Using Eq. (16) and some lengthy algebra, we finally get:

$\rho(\eta)=\frac{M_{\mathrm{vir}}}{4 \pi R_{\mathrm{eff}}^{3} \mathcal{N}_{\mathrm{vir}}} \tilde{\rho}_{1}(\eta) \tilde{\rho}_{2}(\eta)$

with:

$$
\begin{aligned}
\mathcal{N}_{\mathrm{vir}}= & \left(\frac{\eta_{\mathrm{vir}}}{\eta_{0}}\right)^{\alpha}\left(1+\frac{\eta_{\mathrm{vir}}}{\eta_{0}}\right)^{\beta} \gamma\left[n\left(3-p_{n}\right), b_{n} \eta_{\mathrm{vir}}^{1 / n}\right] \\
\tilde{\rho}_{1}= & \left(\frac{\eta}{\eta_{0}}\right)^{\alpha-3}\left(1+\frac{\eta}{\eta_{0}}\right)^{\beta-1} \exp \left(-b_{n} \eta^{1 / n}\right), \\
\tilde{\rho}_{2}= & \left(1+\frac{\eta}{\eta_{0}}\right)\left(b_{n} \eta^{1 / n}\right)^{n\left(3-p_{n}\right)} \\
& +n \alpha\left[1+\left(\frac{\alpha+\beta}{\alpha}\right) \eta\right] \gamma\left[n\left(3-p_{n}\right), b_{n} \eta^{1 / n}\right] \mathrm{e}^{b_{n} \eta^{1 / n}} .
\end{aligned}
$$

It is worth stressing that Eq. (18) reduces to the stellar mass profile (10) setting $\alpha=\beta=0$ and $\Upsilon_{\text {eff }}=\Upsilon_{*}$ as expected, while it is positive everywhere for all other values of the parameters provided the above constraints on $\alpha$ and $\alpha+\beta$ are set. In order to get further insights on the shape of the density profile, one may consider its logarithmic slope:

$s(\eta)=\frac{\mathrm{d} \ln \rho}{\mathrm{d} \ln r}=\frac{\eta}{\rho} \frac{\mathrm{d} \rho}{\mathrm{d} \eta}$.

Some lengthy algebra finally gives:

$s(\eta)=s_{1}(\eta)+s_{2}(\eta)$,

having defined:

$s_{1}(\eta)=\frac{(\alpha-3)+(\alpha+\beta-4)\left(\eta / \eta_{0}\right)}{1+\eta / \eta_{0}}-\left(b_{n} / n\right) \eta^{1 / n}$, $s_{2}(\eta)=\frac{\eta \mathcal{N}_{2}(\eta)}{\eta_{0} \mathcal{D}_{2}(\eta)}$

$$
\begin{aligned}
\mathcal{N}_{2}= & \left(b_{n} \eta^{1 / n}\right)^{n\left(3-p_{n}\right)}+b_{n}^{n\left(3-p_{n}\right)} \eta_{0} \eta^{2-p_{n}} \\
& \times\left\{\left(3-p_{n}\right)\left(1+\eta / \eta_{0}\right)+\alpha\left[1+(\alpha+\beta)\left(\eta / \eta_{0}\right) / \alpha\right]\right\} \\
& +\left\{n(\alpha+\beta)+\alpha \eta_{0}\left[1+(\alpha+\beta)\left(\eta / \eta_{0}\right) / \alpha\right] \eta^{-1+1 / n}\right\} \\
& \times \gamma\left[n\left(3-p_{n}\right), b_{n} \eta^{1 / n}\right], \\
\mathcal{D}_{2}= & \left(b_{n} \eta^{1 / n}\right)^{n\left(3-p_{n}\right)}\left(1+\eta / \eta_{0}\right)+n \alpha \gamma\left[n\left(3-p_{n}\right), b_{n} \eta^{1 / n}\right] \\
& \times\left[1+(\alpha+\beta)\left(\eta+\eta_{0}\right) / \alpha\right] \exp \left(b_{n} \eta^{1 / n}\right) .
\end{aligned}
$$

The density profile may be locally approximated as a power law with a running slope, i.e. we may write $\rho(\eta) \sim \eta^{s(\eta)}$. It is therefore particularly interesting to consider the asymptotic limits of the logarithmic slope. To this aim, we first set $n=4$ to get:

$\lim _{\eta \rightarrow 0} s(\eta, n=4)=\alpha-3$

$\lim _{\eta \rightarrow \infty} s(\eta, n=4)=-\infty$

so that we get the constraint $\alpha \leq 3$ in order that $\rho(\eta)$ is not an increasing function of the radius in the inner regions. Note also that models with $\alpha=3$ provide a density profile with an inner core thus resembling popular cases such as the non singular isothermal sphere. On the other hand, in the asymptotic limit $\eta \rightarrow \infty$, the density profile exponentially fades away. Such a behaviour should not be surprising being trivially a consequence of the exponential decay of the luminosity density profile $j(r)$. Although somewhat extreme, such a shape in the outer regions is not unrealistic. Indeed, recent high resolution $\mathrm{N}$-body simulations (Navarro et al. 2004; Merritt et al. 2006) show that the global mass profile is well approximated by an Einasto model (Einasto 1965; Einasto \& Haud 1989; Cardone et al. 2005) which indeed predicts such an exponential decay $^{2}$. It is worth stressing that the same results are obtained also for other values of $n$ as we have checked numerically (for $n \geq 2$ ) so that the constraint on $\alpha$ can be used in the following analysis.

As a final cautionary remark, we stress that the logarithmic slope $s(\eta)$ in Eq. (22) refers to the density law of the effective model defined by our ansatz for the global $M / L$ ratio. As such, $s(\eta)$ is not the logarithmic slope of the (eventually present) dark halo model. Actually, while in the outer regions the stellar density may be neglected so that $s(\eta)$ may be identified with the $s_{\mathrm{DM}}(\eta)$, in the inner regions things are more involved. In particular, an inner core of the effective density profile, i.e. $s(\eta \rightarrow 0)=0$, does not imply that the corresponding dark halo model is a cored one.

Finally, one of the main outcomes of our analysis is the spherical DM content in the core of galaxies, typically within $1 R_{\text {eff }}$. We define the spherical DM fraction within the radius $r$ as

$f_{\mathrm{DM}}(r)=\frac{M_{\mathrm{DM}}(r)}{M_{\mathrm{DM}}(r)+M_{\star}(r)}=1-\frac{M_{\star}(r)}{M(r)}$

where $M_{\star}(r)=\Upsilon_{*} L(r)$ and $M_{\mathrm{DM}}(r)$ is DM mass.

2 Actually, these are DM only simulations so that the addition of a baryonic component changes the global mass profile. Nevertheless, in the very outer regions, the stellar component may be neglected so that it is likely that the global mass profile behaves as the DM one thus recovering the exponential decay predicted by these simulations. 


\subsection{The surface mass density}

A further quantity to be discussed is the surface mass density, which we compute as:

$\Sigma(R)=2 \int_{0}^{\infty} \rho(r) \mathrm{d} z$

having defined $R=\left(x^{2}+y^{2}\right)^{1 / 2}$ as usual. It is convenient to rewrite the above integral as:

$\Sigma(R)=2 \int_{0}^{\pi / 2} \frac{\rho(R / \sin \theta) R}{\sin ^{2} \theta} \mathrm{d} \theta$

with $r=R / \sin \theta$, so that, inserting Eq. (18) for $\rho(r)$ and defining $\xi=R / R_{\text {eff }}$, after some algebra, we finally get:

$\Sigma(\xi)=\frac{M_{\mathrm{vir}} \xi^{\alpha-2}}{2 \pi n \eta_{0}^{\alpha} R_{\text {eff }}^{2} \mathcal{N}_{\text {vir }}} \tilde{\Sigma}(\xi)=\frac{\Sigma_{\text {eff }}}{\xi^{2-\alpha}} \frac{\tilde{\Sigma}(\xi)}{\tilde{\Sigma}(\xi=1)}$

with:

$$
\begin{aligned}
\tilde{\Sigma}(\xi)= & \int_{0}^{\pi / 2}\left(1+\frac{\xi / \eta_{0}}{\sin \theta}\right)^{\beta-1} \frac{\tilde{\rho}_{2}(\xi / \sin \theta)}{\sin ^{\alpha-1} \theta} \\
& \times \exp \left[-b_{n}\left(\frac{\xi}{\sin \theta}\right)^{1 / n}\right] \mathrm{d} \theta, \\
\Sigma_{\mathrm{eff}}= & \frac{M_{\mathrm{vir}}}{2 \pi R_{\mathrm{eff}}^{2}} \frac{\tilde{\Sigma}(\xi=1)}{n \eta_{0}^{\alpha} \mathcal{N}_{\mathrm{vir}}} \\
= & \frac{\Upsilon_{0} L_{\mathrm{T}}}{2 \pi R_{\mathrm{eff}}^{2}} \frac{\tilde{\Sigma}(\xi=1)}{n \eta_{0}^{\alpha} \Gamma\left[n\left(3-p_{n}\right)\right]} \\
= & \frac{\Upsilon_{\mathrm{eff}} L_{\mathrm{T}}}{2 \pi R_{\mathrm{eff}}^{2}} \frac{\eta_{0}^{\beta} \tilde{\Sigma}(\xi=1)}{n\left(1+\eta_{0}\right)^{\beta} \Gamma\left[n\left(3-p_{n}\right)\right]} .
\end{aligned}
$$

Equation (30) clearly shows the role of the different model parameters. Indeed, while $\left(R_{\mathrm{eff}}, I_{\mathrm{e}}, \Upsilon_{\mathrm{eff}}\right)$ only work as scaling quantities through $\Sigma_{\text {eff }}$, the shape of the surface mass density profile is governed by the Sersic index $n$, the slope parameters $(\alpha, \beta)$ and the transition radius $\eta_{0}$. Ideally, one can therefore put further constraints on $(\alpha, \beta)$ demanding that $\Sigma(\xi)$ is a decreasing function in order to be physically viable. Unfortunately, such a program is not feasible. As Eq. (30) shows, there are two terms determining the shape of $\Sigma(\xi)$. While the first one scales as $\xi^{\alpha-2}$, it is not possible to infer a general scaling rule for $\tilde{\Sigma}(\xi)$ since it has to be evaluated numerically. We have therefore investigated the shape of $\Sigma(\xi)$ by evaluating it numerically for different values of $\left(n, \alpha, \alpha+\beta, \eta_{0}\right)$. It turns out that the surface density is a monotonically decreasing function for $\xi>0.01$ whatever are the values of the model parameters. In the very inner region, however, it is sometimes possible to get a cored profile or an unphysical increasing $\Sigma(\xi)$. Since we are unable to work out any analytical rule to identify these models, we prefer to not put any further constraints on the model parameters. Note, however, that, also in this disturbing circumstances, $\Sigma(\xi)$ is bad behaved only in a very inner region where our phenomenological ansätz may break down. Nevertheless, as we will see later, this has no effect on the estimate of the quantities of interest.

\subsection{Projected mass and DM fraction}

A quite interesting derived quantity, which will be useful when contrasting models against observations, is the projected mass within a circular aperture of radius $R$ given by:

$M_{\text {proj }}(R)=2 \pi \int_{0}^{R} \Sigma\left(R^{\prime}\right) R^{\prime} \mathrm{d} R^{\prime}=2 \pi R_{\text {eff }}^{2} \int_{0}^{\xi} \Sigma\left(\xi^{\prime}\right) \xi^{\prime} \mathrm{d} \xi^{\prime}$ which, for our model, reduces to:

$$
M_{\mathrm{proj}}^{\text {th }}(\xi)=\frac{\Upsilon_{\mathrm{eff}} L_{\mathrm{T}}}{n \Gamma\left[n\left(3-p_{n}\right)\right]}\left(\frac{\eta_{0}}{1+\eta_{0}}\right)^{\beta} \int_{0}^{\xi} \tilde{\Sigma}\left(\xi^{\prime}\right) \xi^{\alpha-1} \mathrm{~d} \xi^{\prime} .
$$

For the Prugniel-Simien model we are adopting for the luminosity component, the projected mass is given by:

$$
M_{\mathrm{proj}}^{\mathrm{PS}}(\xi)=\Upsilon_{*} L_{\mathrm{T}}\left[1-\frac{\Gamma\left(2 n, b_{n} \xi^{1 / n}\right)}{\Gamma(2 n)}\right]
$$

so that the projected DM mass fraction reads:

$$
\begin{aligned}
f_{\mathrm{proj}}(\xi)= & 1-\frac{M_{\mathrm{proj}}^{\mathrm{PS}}(\xi)}{M_{\mathrm{proj}}^{\mathrm{th}}(\xi)} \\
= & 1-\frac{n \Gamma\left[n\left(3-p_{n}\right)\right]}{\Upsilon_{\mathrm{eff}} / \Upsilon_{*}}\left(\frac{1+\eta_{0}}{\eta_{0}}\right)^{\beta} \\
& \times \frac{1-\Gamma\left(2 n, b_{n} \xi^{1 / n}\right) / \Gamma(2 n)}{\int_{0}^{\xi} \tilde{\Sigma}\left(\xi^{\prime}\right) \xi^{\prime \alpha-1} \mathrm{~d} \xi^{\prime}} .
\end{aligned}
$$

It is worth noting that the projected mass fraction is typically higher than the spherical mass fraction $f_{\mathrm{DM}}(r)$. This is a consequence of the integration along the line of sight which is performed in order to evaluate $M_{\text {proj }}(R)$. Indeed, the stellar component contributes mainly to the inner region, while the DM halo extends over a larger part of the integration domain. As a result, $f_{\text {proj }}(r)$ is higher than $f_{\mathrm{DM}}(r)$, with the ratio $f_{\mathrm{DM}}(r) / f_{\text {proj }}(r)$ depending both on $r$ and the concentration of both the stars and dark halo density profiles.

\section{Dynamical and gravitational lensing properties}

While photometric observations probe the light distribution (hence giving constraints on the luminosity density), dynamical observables (e.g., velocity dispersion) and gravitational lensing are excellent probes of the mass profile. In the framework of our phenomenological scenario, we can tell that dynamical and lensing observables make it possible to constrain the global $M / L$ ratio $\Upsilon(r)$. Therefore, it is interesting to derive these main properties for our model.

\subsection{Velocity dispersion}

A widely used probe to constrain the model parameters is represented by the line of sight velocity dispersion luminosity weighted within a circular aperture of radius $R_{\mathrm{ap}}$. This can be easily evaluated as:

$\sigma_{\mathrm{ap}}^{2}=\frac{\int_{0}^{R_{\mathrm{ap}}} I(R) \sigma_{\mathrm{los}}^{2}(R) R \mathrm{~d} R}{\int_{0}^{R_{\mathrm{ap}}} I(R) R \mathrm{~d} R}$

with $\sigma_{\text {los }}(R)$ the velocity dispersion projected along the line of sight. This latter may be found solving the Jeans equation provided an expression for the anisotropy parameter is chosen. In order to not further increase the number of unknown quantities in the problem, we assume isotropy in the velocity space. In this case, it is possible to arrive at the following expression for $\sigma_{\text {ap }}$ (Mamon \& Lokas 2005a):

$$
\begin{aligned}
\sigma_{\mathrm{ap}}^{2}= & \frac{4 \pi G}{3 L_{2}\left(R_{\mathrm{ap}}\right)}\left[\int_{0}^{\infty} r j(r) M(r) \mathrm{d} r\right. \\
& \left.-\int_{R_{\mathrm{ap}}}^{\infty}\left(r^{2}-R_{\mathrm{ap}}^{2}\right)^{3 / 2} j(r) M(r) r^{-2} \mathrm{~d} r\right],
\end{aligned}
$$


with

$L_{2}(R)=\int_{0}^{R} I\left(R^{\prime}\right) R^{\prime} \mathrm{d} R^{\prime}$

the projected luminosity profile. For the model we are considering, after some algebra, we finally get:

$$
\begin{aligned}
\sigma_{\mathrm{ap}, \mathrm{th}}^{2}= & \frac{G \Upsilon_{0} L_{\mathrm{T}}}{R_{\mathrm{eff}}} \frac{b_{n}^{n\left(3-p_{n}\right)} \Gamma(2 n)}{3 n \Gamma\left[n\left(3-p_{n}\right)\right]} \frac{\mathcal{I}_{\mathrm{ap}}\left(\xi_{\mathrm{ap}}, \mathbf{p}\right)}{\gamma\left(2 n, b_{n} \xi_{\mathrm{ap}}^{1 / n}\right)} \\
= & \frac{G \Upsilon_{\mathrm{eff}} L_{\mathrm{T}}}{R_{\mathrm{eff}}} \frac{b_{n}^{n\left(3-p_{n}\right)} \Gamma(2 n)}{3 n \Gamma\left[n\left(3-p_{n}\right)\right]}\left(\frac{\eta_{0}^{\alpha}}{1+\eta_{0}}\right)^{\beta} \\
& \times \frac{\mathcal{I}_{\mathrm{ap}}\left(\xi_{\mathrm{ap}}, \mathbf{p}\right)}{\gamma\left(2 n, b_{n} \xi_{\mathrm{ap}}^{1 / n}\right)},
\end{aligned}
$$

where we set $\xi_{\text {ap }}=R_{\text {ap }} / R_{\text {eff }}$, being $R_{\text {ap }}=1.5 \operatorname{arcsec}$ the aperture radius (equal for all lenses because $\sigma_{\text {ap }}$ is measured with the same instrumental setup) and have defined:

$\mathcal{I}_{\mathrm{ap}}=\mathcal{I}_{\mathrm{ap}}^{(1)}-\mathcal{I}_{\mathrm{ap}}^{(2)}$,

$$
\begin{aligned}
\mathcal{I}_{\mathrm{ap}}^{(1)}= & \int_{0}^{\infty}\left(\frac{\eta}{\eta_{0}}\right)^{\alpha}\left(1+\frac{\eta}{\eta_{0}}\right)^{\beta}\left\{1-\frac{\Gamma\left[n\left(3-p_{n}\right), b_{n} \eta^{1 / n}\right]}{\Gamma\left[n\left(3-p_{n}\right)\right]}\right\} \\
& \times \exp \left(-b_{n} \eta^{1 / n}\right) \eta^{1-p_{n}} \mathrm{~d} \eta,
\end{aligned}
$$

$$
\begin{aligned}
\mathcal{I}_{\text {ap }}^{(2)}= & \int_{\xi_{\text {ap }}}^{\infty}\left(\frac{\eta}{\eta_{0}}\right)^{\alpha}\left(1+\frac{\eta}{\eta_{0}}\right)^{\beta}\left\{1-\frac{\Gamma\left[n\left(3-p_{n}\right), b_{n} \eta^{1 / n}\right]}{\Gamma\left[n\left(3-p_{n}\right)\right]}\right\} \\
& \times \exp \left(-b_{n} \eta^{1 / n}\right)\left(\eta^{2}-\xi_{\text {ap }}^{2}\right)^{3 / 2} \eta^{-\left(2+p_{n}\right)} \mathrm{d} \eta .
\end{aligned}
$$

For given values of the model parameters, Eq. (38) allows us to predict the value of $\sigma_{\text {ap }}$. However, because of the uncertaintits on both the photometric parameters and the stellar $M / L$ ratio, the predicted $\sigma_{\text {ap }}$ will be affected by an uncertainty which we compute by the simple propagation of errors. We do not report here the final expression for sake of shortness, but caution the reader that the uncertainty on $R_{\text {eff }}$ must be propagated also in the denominator term $\gamma\left(2 n, b_{n} \xi_{\text {ap }}^{1 / n}\right)$, while we neglect the contribution to the integral for simplicity.

\subsection{Lensing observables}

Gravitational lensing is a powerful tool to further constrain the space of parameters. After having determined the surface mass density in the previous section, here, we define the deflection angle, that allows a model independent estimate of projected mass at Einstein radius to be determined.

\subsubsection{The deflection angle}

A key role in the determination of the lensing properties of a given model is played by the deflection angle. For a spherically symmetric lens, this reads (Schneider et al. 1992):

$\hat{\alpha}=\frac{2}{R} \int_{0}^{R} \frac{\Sigma\left(R^{\prime}\right)}{\Sigma_{\text {crit }}} R^{\prime} \mathrm{d} R^{\prime}$

where the critical surface density $\Sigma_{\text {crit }}$ is:

$\Sigma_{\text {crit }}=\frac{c^{2} D_{\mathrm{s}}}{4 \pi G D_{\mathrm{L}} D_{\mathrm{LS}}}$ with $D_{\mathrm{S}}, D_{\mathrm{L}}, D_{\mathrm{LS}}$ the observer-source, observer-lens and lens-source comoving angular diameter distances, respectively. Inserting Eq. (30), the deflection angle for our model is easily computed as:

$$
\begin{aligned}
\hat{\alpha}(\xi) & =\frac{2 R_{\mathrm{eff}}}{\tilde{\Sigma}(\xi=1)} \frac{\Sigma_{\mathrm{eff}}}{\Sigma_{\text {crit }}} \frac{1}{\xi} \int_{0}^{\xi} \tilde{\Sigma}\left(\xi^{\prime}\right) \xi^{\alpha^{\alpha-1}} \mathrm{~d} \xi^{\prime} \\
& =\frac{\hat{\alpha}_{\mathrm{eff}}}{\xi} \frac{\tilde{\alpha}(\xi)}{\tilde{\alpha}(\xi=1)}
\end{aligned}
$$

where we have defined ${ }^{3}$ :

$$
\begin{aligned}
\tilde{\alpha}(\xi) & =\int_{0}^{\xi} \xi^{\alpha-1} \tilde{\Sigma}\left(\xi^{\prime}\right) \mathrm{d} \xi^{\prime} \\
\hat{\alpha}_{\mathrm{eff}} & =2 R_{\mathrm{eff}} \frac{\Sigma_{\mathrm{eff}}}{\Sigma_{\mathrm{crit}}} \frac{\tilde{\alpha}(\xi=1)}{\tilde{\Sigma}(\xi=1)} \\
& =2 R_{\mathrm{eff}} \frac{\Upsilon_{0} L_{\mathrm{T}}}{2 \pi \Sigma_{\text {crit }} R_{\mathrm{eff}}^{2}} \frac{\tilde{\alpha}(\xi=1)}{n \eta_{0}^{\alpha} \Gamma\left[n\left(3-p_{n}\right)\right]} \\
& =2 R_{\mathrm{eff}} \frac{\Upsilon_{\mathrm{eff}} L_{\mathrm{T}}}{2 \pi \Sigma_{\mathrm{crit}} R_{\mathrm{eff}}^{2}} \frac{\eta_{0}^{\beta} \tilde{\alpha}(\xi=1)}{n\left(1+\eta_{0}\right)^{\beta} \Gamma\left[n\left(3-p_{n}\right)\right]}
\end{aligned}
$$

Looking at Eqs. (42) and (44), it is immediately clear that the Sersic index $n$ and the model parameters $\left(\alpha, \beta, \eta_{0}\right)$ determine the shape of the deflection angle, while the effective radius $R_{\text {eff }}$, the total luminosity $L_{\mathrm{T}}$ and the mass parameter $\Upsilon_{\mathrm{eff}}$ only work as scaling quantities.

\subsubsection{The Einstein angle}

Should the source be a distant pointlike object (such as a quasar), one may observe multiple images and then use their positions to constrain the model parameters. On the other hand, when the source is an extended object approximately aligned with the lens along the line of sight, the formation of arcs takes place. The angular radius of the circle individuated by these arcs is the Einstein angle $R_{\mathrm{E}}$. Referring the interested reader to the literature for more details, we here only remember that the Einstein angle may be determined by solving:

$\hat{\alpha}\left(\xi_{\mathrm{E}}\right)=R_{\mathrm{E}}=\xi_{\mathrm{E}} R_{\mathrm{eff}}$,

with $^{4} \xi_{\mathrm{E}}=R_{\mathrm{E}} / R_{\mathrm{eff}}$, so that a measurement of $R_{\mathrm{E}}$ (in $\operatorname{arcsec}$ ) provides a constraint on the model parameters through the deflection angle $\hat{\alpha}\left(\xi_{\mathrm{E}}\right)$. Moreover, comparing the definition of $M_{\text {proj }}(\xi)$ and $\hat{\alpha}(\xi)$, it is immediate to get:

$M_{\text {proj }}\left(R_{\mathrm{E}}\right)=\pi R_{\mathrm{E}}^{2} \Sigma_{\text {crit }}=\pi \xi_{\mathrm{E}} R_{\text {eff }}^{2} \Sigma_{\text {crit }}$

with $R_{\text {eff }}$ now expressed in linear units. Eq. (46) allows then to estimate the projected mass within the Einstein radius provided that $\Sigma_{\text {crit }}$ is known, i.e., the lens and source redshift have been measured.

\footnotetext{
${ }_{3}$ A dimensional remark is in order here. The deflection angle is expressed in $\operatorname{arcsec}$ so that, although the effective radius $R_{\text {eff }}$ is a length, in the right hand side of Eq. (42), it must be expressed in angular units. ${ }^{4}$ Here, we recast the definition for deflection angle and projected mass introducing the adimensional radius $\xi_{E}$.
} 


\section{Testing the model global $M / L$ ratio}

Our proposed parametrization for $\Upsilon(r)$ was motivated by the need to have a versatile expression able to mimic a large set of galaxy models. Since we assume that the stellar component is fixed and described by a PS density profile, mimicking a large set of models is the same as mimicking the global $M / L$ ratio predicted by different dark halo models. Needless to say, such an ambitious goal is difficult to reach so that we expect that Eq. (1) does a good job over a limited radial range. It is therefore worth exploring how large is this range and how good and versatile is our approximated parametrization.

To this aim, it is convenient to resort to the following general expression for the mass profile of the dark halo:

$M_{\mathrm{DM}}(r)=M_{\mathrm{vir}} \times \mu\left(r / r_{\mathrm{s}}\right)=M_{\mathrm{vir}} \times \mu\left(\eta / \eta_{\mathrm{s}}\right)$

with $M_{\text {vir }}$ the virial mass and $r_{\mathrm{s}}=R_{\mathrm{eff}} \eta_{\mathrm{s}}$ a scalelength. As a function of the adimensional length $\eta$ the DM mass fraction then reads:

$f_{\mathrm{DM}}(\eta)=\left[1+\frac{M_{\star}(\eta)}{M_{\mathrm{DM}}(\eta)}\right]^{-1}$.

Normalizing with respect to $f_{\mathrm{e}}=f_{\mathrm{DM}}\left(R=R_{\text {eff }}\right)$ and using Eq. (12), we finally get:

$$
\begin{aligned}
f_{\mathrm{DM}}(\eta) & =\left\{1+\frac{1-f_{\mathrm{e}}}{f_{\mathrm{e}}} \frac{\mu\left(1 / \eta_{\mathrm{s}}\right)}{\mu(\eta)}\right. \\
& \left.\times \frac{1+\Gamma\left[n\left(3-p_{n}\right), b_{n} \eta^{1 / n}\right] / \Gamma\left[n\left(3-p_{n}\right)\right]}{1+\Gamma\left[n\left(3-p_{n}\right), b_{n}\right] / \Gamma\left[n\left(3-p_{n}\right)\right]}\right\}^{-1}
\end{aligned}
$$

which may then be related to the global $M / L$ ratio as:

$\Upsilon(\eta)=\frac{\Upsilon_{*}}{1-f_{\mathrm{DM}}(\eta)}$

having assumed a constant stellar $M / L$ ratio. Eq. (47) makes it possible to compute the DM mass fraction and hence the global $M / L$ ratio through Eq. (48) provided that an expression for $\mu\left(\eta / \eta_{\mathrm{s}}\right)$ is given.

To test the validity of our phenomenological ansatz, we start considering the most popular galaxy profiles, i.e. NFW (Navarro et al. 1996, 1997), the Einasto (Einasto 1965; Einasto \& Haud 1989; $\quad$ Navarro et al. 2004; Cardone et al. 2005), the nonsingular isothermal sphere and the Burkert (1995) models. Using the formalism introduced above, the cumulative mass profile for each case is assigned by the following expressions:

$\mu_{\mathrm{NFW}}\left(\eta / \eta_{\mathrm{s}}\right)=\log \left(1+\eta / \eta_{\mathrm{s}}\right)-\left(\eta / \eta_{\mathrm{s}}\right)\left(1+\eta / \eta_{\mathrm{s}}\right)^{-1}$,

$\mu_{\text {Ein }}(\eta)=1-\frac{\Gamma\left[2 n_{\mathrm{DM}}, \mathrm{d}_{n}\left(\eta / \eta_{\mathrm{s}}\right)^{1 / n_{\mathrm{DM}}}\right]}{\Gamma\left(2 n_{\mathrm{DM}}\right)}$,

$\mu_{\mathrm{NIS}}(\eta)=\eta / \eta_{\mathrm{s}}-\arctan \left(\eta / \eta_{\mathrm{s}}\right)$,

$\mu_{\mathrm{Bur}}(\eta)=\frac{\ln \left[1+\left(\eta / \eta_{\mathrm{s}}\right)^{2}\right]}{4}+\frac{\ln \left[1+\left(\eta / \eta_{\mathrm{s}}\right)\right]-\arctan \left(\eta / \eta_{\mathrm{s}}\right)}{2}$.

We parameterize NFW profile assigning first the virial mass $M_{\mathrm{vir}}$ and then estimating the scalelength $r_{\mathrm{s}}$ as $r_{\mathrm{s}}=R_{\mathrm{vir}} / c$ using the following numerically motivated relation (Bullock et al. 2001; Napolitano et al. 2005) to set the concentration parameter:

$c=\frac{16.7}{1+z}\left(\frac{M_{\mathrm{vir}}}{10^{11} h^{-1} M_{\odot}}\right)^{-0.125}$ also taking care of the large scatter. It is worth noting that, by virtue of the $c-M_{\mathrm{vir}}$ relation, the NFW profile reduces to a oneparameter model. In contrast, since no numerically or observationally motivated relation between $\left(n_{\mathrm{DM}}, \eta_{\mathrm{s}}, M_{\mathrm{vir}}\right)$ is known, the Einasto model is assigned by three parameters that we choose in such a way to have a realistic density profile. A similar discussion also holds for both the NIS and Burkert models which are, however, two - parameters profiles. As a further remark, we stress that, while the NIS and Burkert models possess an inner core, the NFW and Einasto models are cuspy even if with different logarithmic slope for $\eta \ll \eta_{\mathrm{s}}$. Moreover, the outer slope of the density profile runs from $s=-2$ for the NIS model, to $s=-3$ for both NFW and Burkert cases, to $s=-\infty$ (i.e. exponential decay) for the Einasto parametrization. As such, by considering these four models, we are spanning a wide range of possibilities thus allowing us to test how good our phenomenological proposal for the global $M / L$ ratio $\Upsilon(r)$ mimics a large class of different profiles.

Once the halo parameters have been set, we add a stellar component fixing the photometric parameters $\left(n, R_{\mathrm{eff}}, I_{\mathrm{e}}\right)$, the stellar $M / L$ ratio $\Upsilon_{*}$ and the redshift $z$ so that they are equal to those of a randomly chosen lens. We then fit Eq. (1) to the resulting $\Upsilon(\eta)$ profile and repeat this exercise for 1000 random realizations of each halo model. The instructive results of this investigation are schematically summarized below.

- Equation (1) fits the $\Upsilon(r)$ of the tested models with a very good precision and over a large radial range, namely $0.01 \leq$ $\eta \leq 10$. Denoting with $\Upsilon_{\text {fit }}$ our proposed function and defining $\Delta=\left|1-\Upsilon_{\text {fit }} / \Upsilon\right|$, we get that the maximal deviation is lower than $15 \%$ (depending on the model). In particular, for NFW is $0.06 \% \leq \Delta_{\text {rms }} \leq 1.8 \%$ and $2 \% \leq \Delta_{\max } \leq 8 \%$. The Einasto model is fitted with $\Delta_{\mathrm{rms}} \sim 0.5 \%$, while the worst performances are obtained for the Burkert one for which we, however, find a still satisfactory $\Delta_{\mathrm{rms}} \sim 6 \%$.

- The fitted model parameters $\left(\alpha, \beta, \eta_{0}, \Upsilon_{\mathrm{eff}} / \Upsilon_{*}\right)$ depend on the halo parameters so that a general rule cannot be given. However, we note that quite small and negative values of $\alpha$ are clearly preferred for the NFW+PS input model. This is somewhat surprising since this would give rise to models with an inner asymptotic slope of the global density $s_{0}=\alpha-3<-3$, contrary to popular models having $s_{0} \simeq-1$. However, as stated above, in the very inner regions our model does not fit anymore the NFW + PS one so that the results on $s_{0}$ can not be trusted upon. For this same reason, we do not care about obtaining still more negative values of $\alpha$. Indeed, for the other models, $\alpha<0$ cases are still clearly preferred. As a conservative estimate, we therefore conclude that, in order our parametrization fits well different dark halo models, one must have negative $\alpha$, with values as low as $\alpha \simeq-0.40$.

- The asymptotic outer slope $\alpha+\beta$ typically takes values of order $1-2$, even if one cannot exclude values as high as $\alpha+\beta \simeq 5$. For example, if considering the NFW $+\mathrm{PS}, \alpha+\beta$ is strongly peaked on the $\sim 2$, and the distributions is markedly asymmetric with long tails towards larger values. In contrast, $\alpha+\beta$ has a similar distribution, but shifted to the smaller $\alpha+\beta \simeq 0.5$ peak, for cored models. As a general rule, however, $\alpha+\beta \leq 0$ never occurs in agreement with our above discussion showing that such models have an unphysically decreasing mass profile.

- The scaling quantities $\Upsilon_{\text {eff }} / \Upsilon_{*}$ and $\log \eta_{0}$ are in the range $1.0 \leq \Upsilon_{\text {eff }} / \Upsilon_{*} \leq 2.5$ and $-0.5 \leq \log \eta_{0} \leq 1.5$. In particular, if considering the NFW $+\mathrm{PS}, \Upsilon_{\text {eff }} / \Upsilon_{*}$ and $\log \eta_{0}$ are strongly peaked on $\sim 1.5$ and 0.5 respectively. Similar values are 
obtained for the other dark halo models considered, a result which is not fully unexpected. Indeed, $\Upsilon_{\text {eff }} / \Upsilon_{\star}$ and $\log \eta_{0}$ are mostly related respectively to the virial mass $M_{\text {vir }}$ and DM mass fration $f_{\mathrm{e}}$ and to the scaled virial radii $R_{\mathrm{vir}} / R_{\mathrm{eff}}$. Since these quantities take similar values for all models, we indeed expect to find a not too large scatter among different models which is indeed what we find.

- Changing the $c-M_{\text {vir }}$ relation or its scatter does not alter the result on the validity of our approximation for NFW + PS profile, i.e. $\Delta_{\mathrm{rms}}$ and $\Delta_{\max }$ span the same range as above for $\eta$ covering the same radial range. However, the distribution of the fitted parameters may change significantly. In particular, higher values of $\alpha$ may be obtained although negative $\alpha$ are still preferred ${ }^{5}$. Considering that similar values for $\alpha$ can be obtained by fitting $\Upsilon_{\text {fit }}$ to models other than NFW, we argue that it is not possible to resort to $\alpha$ to discriminate between, e.g., the NFW and the Einasto models, or cusped and cored profiles, unless one has a precise determination of the $c-M_{\mathrm{vir}}$ relation.

These results clearly show that our proposed parametrization of the global $M / L$ ratio indeed makes very well its job. It is indeed able to reproduce with very good accuracy the expected behaviour of $M(r) / L(r)$ whatever is the dark halo density profile over a radial range spanning three orders of magnitude. As a final cautionary test, we check that using this very good approximation does not introduce any bias in the lensing deflection angle. As an example, we can consider the case of the NFW model and comparing the value of $\hat{\alpha}(\xi)$ computed using the analytical NFW deflection angle and the numerically evaluated Eq. (42). We repeat this test for 1000 random realizations and estimate the ratio $\hat{\alpha}_{\mathrm{NFW}}(\xi) / \hat{\alpha}_{\mathrm{fit}}(\xi)$ for $\xi=1$ and $\xi=\xi_{\mathrm{E}}$, using for $\xi_{\mathrm{E}}$ the observational value for the lens used to set the stellar parameters. It turns out that both these ratios deviate from unity less than $1 \%$ confirming our expectation that no bias is induced by the use of our ansatz.

\section{Model vs observations}

The phenomenological ansatz for $\Upsilon(r)$ we are proposing is characterized by four parameters, namely the two asymptotic slopes $^{6}$, the logarithm of the scaling length $\log \eta_{0}$ and the global $M / L$ ratio at the effective radius $\Upsilon_{\text {eff. }}$. To these four quantities, we have to add the stellar $M / L$ ratio $\Upsilon_{*}$ thus leading to five the number of unknown quantities to be constrained. Needless to say, tackling such an issue is a quite daunting task. A possible way out could be contrasting the model with kinematic observations, such as the velocity dispersion profile. To this aim, the data should cover a radial range wide enough to sample with sufficient detail both the inner and the outer regions in order to constrain the two asymptotic slopes. Moreover, the measurement errors and the sampling should be extremely good to overcome

\footnotetext{
${ }^{5}$ Note that the relation between the concentration $c$ and $\alpha$ is difficult to extract. Indeed, since we set $\alpha$ as a result of fitting our phenomenological model to an input stellar plus dark halo profile, its value also depends on the fitted values of the other parameters, $\left(\alpha+\beta, \eta_{0}\right)$. As a result, we are therefore unable to say whether a larger $\alpha$ implies a higher or smaller concentration.

${ }^{6}$ Hereafter, we will consider $\alpha+\beta$ rather than $\beta$ as model parameter since it is this former quantity that sets the asymptotic slope of the global $M / L$ ratio. Moreover, we have physically motivated constraints on $\alpha$ and $\alpha+\beta$ so that the ranges to be explored for these parameters is immediately set. We also change from $\eta_{0}$ to $\log \eta_{0}$ as scalength parameter in order to explore a wider range for this quantity.
}

the problem of parameters degeneracies in a five dimensional space. All these observational requirements are quite demanding so as to be satisfied only by a handful number of nearby galaxies. Actually, we are interested here in lens galaxies with typical redshifts of order $0.1-0.5$ so that measuring their velocity dispersion profile with the above precision and sampling is an unrealistic task. Nevertheless, for each galaxy, we still have two observable quantities that can be used, namely the Einstein angle $R_{\mathrm{E}}$ and $\sigma_{\mathrm{ap}}$, the line of sight velocity dispersion luminosity weighted in a circular aperture of radius $R_{\mathrm{ap}}$. Needless to say, it is impossible to constraint a five dimensional parameter space with only two datapoints so that a case-by-case analysis is not possible. In order to overcome such a problem, we have therefore to reduce the number of unknowns and increase the number of observed datapoints. To this aim, we therefore first get an estimate of the stellar $M / L$ ratio $\Upsilon_{*}$.

\subsection{Stellar $M / L$}

We start assembling a library of synthetic stellar population models obtained through the Galaxev code (Bruzual \& Charlot 2003) varying the age of the population, its metallicity and time lag of the exponential star formation rate and assuming a Chabrier (2001) initial mass function (IMF). Then, we use the tabulated $(u, g, r, i, z)$ apparent magnitudes (corrected for extinction) of each lens to fit the above library of spectra (suitably redshifted to lens redshift) to the colours, thus getting the estimates reported in Table 1. Note that these values may be easily scaled to a Salpeter (1955) or Kroupa (2001) IMF by multiplying by 1.8 or 1.125 respectively so that we can explore other IMF choices ${ }^{7}$. In order to get the $\Upsilon_{*}$ uncertainties, we use a Monte Carlo-like procedure generating a set of colours from a Gaussian distribution centred on each mean colour and standard deviation equal to the colour uncertainty. Fitting the colours thus obtained to the synthetic spectra for each realization, we generate a distribution of fitted parameters. The median and median scatter of such a distribution is finally taken as an estimate of $\Upsilon_{*}$ and its uncertainty (see Tortora et al. 2009, for further details).

\subsection{Resorting to universal parameters}

Having reduced by one the number of parameters, we now look for a way to increase the number of constraints. To this aim, we could stack all the lenses in a single sample. However, while one can reasonably argue that the same functional expression for $\Upsilon(r)$ describes the global $M / L$ ratio of all the lenses, its characterizing parameters are likely to change on a case-by-case basis. Indeed, even if we assume that the DM halo has a universal profile, the details of the baryonic assembly may lead to different model parameters. In order to partially alleviate this problem, we therefore reparametrize $\Upsilon(r)$ in Eq. (1) in terms of quantities that are likely to be (at least, to first order) universal.

To this aim, it is worth remembering that previous works on fitting galaxy models to the lenses using the constraints from the Einstein ring and the velocity dispersion concordantly suggest that the total mass profile at $R=R_{\mathrm{E}}$ is well approximated by a singular isothermal sphere (see, e.g., Treu \& Koopmans 2004, and refs. therein). Actually, rather than constraining the global

\footnotetext{
7 While this is correct for a Salpeter IMF, since it differs from a Chabrier IMF only for the low mass slope and predict very similar colours, for a Kroupa IMF this is not strictly true, but we could assume the scale factor above as a good approximation.
} 
Table 1. Photometric and lensing observables and estimated stellar $M / L$ ratio for the 21 SLACS lenses.

\begin{tabular}{ccccccccc}
\hline \hline Name & $z_{L}$ & $z_{S}$ & $M_{V}$ & $R_{\text {eff }}$ & $R_{\mathrm{E}}$ & $\sigma_{\text {ap }}$ & $R_{\text {ap }} / R_{\text {eff }}$ & $\Upsilon_{*}$ \\
\hline J002907.8-005550 & 0.2270 & 0.9313 & -21.53 & 1.48 & 0.82 & $229 \pm 18$ & 1.01 & $1.85 \pm 0.15$ \\
J015758.9-005626 & 0.5132 & 0.9243 & -22.16 & 0.93 & 0.72 & $295 \pm 47$ & 1.61 & $2.00 \pm 0.45$ \\
J021652.5-081345 & 0.3317 & 0.5235 & -22.95 & 2.79 & 1.15 & $333 \pm 23$ & 0.54 & $2.12 \pm 0.09$ \\
$\mathrm{~J} 025245.2+00358$ & 0.2803 & 0.9818 & -21.67 & 1.69 & 0.98 & $164 \pm 12$ & 0.89 & $2.19 \pm 0.29$ \\
$\mathrm{~J} 033012.1-002052$ & 0.3507 & 1.0709 & -21.86 & 1.17 & 1.06 & $212 \pm 21$ & 1.28 & $2.49 \pm 0.15$ \\
$\mathrm{~J} 072805.0+383526$ & 0.2058 & 0.6877 & -21.80 & 1.33 & 1.25 & $214 \pm 11$ & 1.13 & $1.80 \pm 0.15$ \\
$\mathrm{~J} 080858.8+470639$ & 0.2195 & 1.0251 & -21.77 & 1.65 & 1.23 & $236 \pm 11$ & 0.91 & $2.20 \pm 0.15$ \\
$\mathrm{~J} 090315.2+411609$ & 0.4304 & 1.0645 & -22.25 & 1.28 & 1.13 & $223 \pm 27$ & 1.17 & $2.09 \pm 0.15$ \\
$\mathrm{~J} 091205.3+002901$ & 0.1642 & 0.3239 & -22.95 & 5.50 & 1.61 & $326 \pm 16$ & 0.27 & $1.45 \pm 0.07$ \\
$\mathrm{~J} 095944.1+041017$ & 0.1260 & 0.5350 & -20.94 & 1.99 & 1.00 & $197 \pm 13$ & 0.75 & $2.09 \pm 0.10$ \\
$\mathrm{~J} 102332.3+423002$ & 0.1912 & 0.6960 & -21.56 & 1.40 & 1.30 & $242 \pm 15$ & 1.07 & $2.54 \pm 0.09$ \\
$\mathrm{~J} 110308.2+532228$ & 0.1582 & 0.7353 & -22.02 & 3.22 & 0.84 & $196 \pm 12$ & 0.46 & $1.10 \pm 0.06$ \\
$\mathrm{~J} 120540.4+491029$ & 0.2150 & 0.4808 & -22.00 & 1.92 & 1.04 & $281 \pm 14$ & 0.78 & $1.98 \pm 0.16$ \\
$\mathrm{~J} 125028.3+052349$ & 0.2318 & 0.7953 & -22.17 & 1.64 & 1.15 & $252 \pm 14$ & 0.91 & $2.18 \pm 0.10$ \\
$\mathrm{~J} 140228.1+632133$ & 0.2046 & 0.4814 & -22.20 & 2.29 & 1.39 & $267 \pm 17$ & 0.65 & $1.50 \pm 0.08$ \\
$\mathrm{~J} 142015.9+601915$ & 0.0629 & 0.5351 & -21.04 & 2.49 & 1.04 & $205 \pm 10$ & 0.60 & $1.24 \pm 0.10$ \\
$\mathrm{~J} 162746.5-005358$ & 0.2076 & 0.5241 & -22.06 & 2.47 & 1.21 & $290 \pm 15$ & 0.61 & $1.80 \pm 0.89$ \\
$\mathrm{~J} 163028.2+452036$ & 0.2479 & 0.7933 & -22.31 & 2.01 & 1.81 & $276 \pm 16$ & 0.75 & $2.42 \pm 0.09$ \\
$\mathrm{~J} 230053.2+002238$ & 0.2285 & 0.4635 & -22.06 & 2.22 & 1.25 & $279 \pm 17$ & 0.68 & $1.98 \pm 0.16$ \\
$\mathrm{~J} 230321.7+142218$ & 0.1553 & 0.5170 & -22.40 & 3.73 & 1.64 & $255 \pm 16$ & 0.40 & $2.49 \pm 0.08$ \\
$\mathrm{~J} 234111.6+000019$ & 0.1860 & 0.8070 & -22.14 & 3.20 & 1.28 & $207 \pm 13$ & 0.47 & $2.89 \pm 0.15$ \\
\hline
\end{tabular}

mass profile, such an analysis essentially probe the shape of the density profile only at the Einstein radius so that one can argue that the logarithmic slope of the density profile at $R_{\mathrm{E}} / R_{\mathrm{eff}}$ is the same for all lenses. Motivated by these previous literature results, we therefore assume that $s_{\mathrm{E}}=s\left(R_{\mathrm{E}} / R_{\text {eff }}\right)$ is the same for all lenses and use this quantity as model parameter instead of $\log \eta_{0}$. To this aim, for given values of $\left(\alpha, \alpha+\beta, s_{\mathrm{E}}\right)$, we numerically solve:

$s\left(R_{\mathrm{E}} / R_{\mathrm{eff}}\right)=s_{\mathrm{E}}$

with respect to $\log \eta_{0}$ using Eqs. (22)-(26) to compute $s(\eta)$. We then note that the DM mass fraction at the virial radius is likely to be a universal quantity. Indeed, considering that:

$f_{\mathrm{vir}}=1-\frac{M_{\star}\left(R_{\mathrm{vir}}\right)}{M_{\mathrm{vir}}} \simeq 1-\frac{\Upsilon_{*} L_{\mathrm{T}}}{M_{\mathrm{vir}}}$,

and setting $M_{\star}\left(R_{\mathrm{vir}}\right) / M_{\mathrm{vir}}=\varepsilon_{\mathrm{SF}} f_{b}$ with $f_{\mathrm{b}}=\Omega_{\mathrm{b}} / \Omega_{\mathrm{M}}$ the cosmic baryon fraction (Spergel et al. 2003) and $\varepsilon_{\mathrm{SF}}$ the star formation efficiency, we can assume that, notwithstanding the details of the star formation process, $\varepsilon_{\mathrm{SF}}$ is roughly the same for all the lenses in the sample. Although not strictly true, this is, however, a resonable approximation since all the lenses we are considering are elliptical galaxies spanning a limited range in both redshift and luminosity. As such, we will hereafter assume that $\mu_{\mathrm{vir}}=M_{\mathrm{vir}} / \Upsilon_{*} L_{\mathrm{T}}$ is a universal quantity to set the $\Upsilon_{\text {eff }}$ parameter as:

$\Upsilon_{\mathrm{eff}}=\frac{M_{\mathrm{vir}}}{\eta_{0}^{\alpha} L_{\mathrm{T}}}\left(\frac{1+\eta_{0}}{\eta_{\mathrm{vir}}+\eta_{0}}\right)^{\beta} \frac{\Gamma\left[n\left(3-p_{n}\right)\right]}{\gamma\left[n\left(3-p_{n}\right), b_{n} \eta_{\mathrm{vir}}^{1 / n}\right]}$

with $M_{\mathrm{vir}}=\mu_{\mathrm{vir}} \Upsilon_{*} L_{\mathrm{T}}$. Note that, here, we are assuming that $M_{\star}\left(R_{\mathrm{vir}}\right)$ is the total stellar mass $\Upsilon_{*} L_{\mathrm{T}}$ which, although not strictly true, is a very good approximation.

Unfortunately, we are unable to find other lens related parameters that can be considered universal so that we will hereafter parameterize our $\Upsilon(r)$ ansatz with the slope parameters $\alpha$ and $\alpha+\beta$, the logarithmic slope at the scaled Eintein radius $s_{\mathrm{E}}=s\left(R_{\mathrm{E}} / R_{\mathrm{eff}}\right)$, and the mass ratio at the virial radius $\mu_{\mathrm{vir}}=M_{\mathrm{vir}} / \Upsilon_{*} L_{\mathrm{T}}$ assuming that these are universal quantities.
In order to explore the impact of this theoretical hypothesis, we will fit our model to the full lens sample and to four subsamples containing respectively five, six, six, four lenses binned according to the absolute $V$ magnitude $M_{V}$. Comparing the constraints on the model parameters obtained by the different fits makes it possible to look for an eventual dependence on the lens luminosity thus giving an a posteriori check of our a priori assumption.

\subsection{Statistical analysis}

As shown by Eq. (45), a measurement of $\xi_{\mathrm{E}}$ may be considered as a measurement of the deflection angle $\hat{\alpha}\left(\xi_{\mathrm{E}}\right)$ so that stacking together many galaxies with different values of $\xi_{\mathrm{E}}$ makes it possible to reconstruct the deflection angle profile. Similarly, since $\sigma_{\text {ap }}$ is weighted within an aperture of fixed angular radius $R_{\text {ap }}$ but different normalized radii $\xi_{\text {ap }}=R_{\text {ap }} / R_{\text {eff }}$, this is the same as tracing the luminosity weighted line of sight dispersion profile. The agreement within the model and the data may then be optimized by maximizing the likelihood function:

$\mathcal{L}(\mathbf{p}) \propto \exp \left[-\frac{\chi_{\text {lens }}^{2}(\mathbf{p})+\chi_{\text {dyn }}^{2}(\mathbf{p})}{2}\right]$

with $\mathbf{p}=\left(\alpha, \alpha+\beta, s_{\mathrm{E}}, \mu_{\mathrm{vir}}\right)$ the set of model parameters and $\chi_{\text {lens }}^{2}\left(\chi_{\text {dyn }}^{2}\right)$ a merit function for the lensing (dynamics) related observables. Maximizing $\mathcal{L}(\mathbf{p})$ gives us the best fit model parameters, while the isolikelihood contours provide constraints on the parameter space that are difficult to quantify because of the four dimensional nature of this space. In order to carry out the maximization of $\mathcal{L}(\mathbf{p})$, we implement a Markov Chain Monte Carlo algorithm which efficiently samples the regions of high likelihood. The points in the chain may be also used to extract constraints on a given parameter $p_{i}$ marginalizing over the other ones. To this aim, we consider the histogram of the $p_{i}$ values along the chain and, following a Bayesian approach, take the median of this histogram as the best estimate of the parameter $p_{i}$, while 68 and $95 \%$ quantiles of this distribution give us the 68 and $95 \%$ confidence ranges for the parameter $p_{i}$. Note that, since the distributions may also be not Gaussian, the median and the mean value may be different, but we still retain the median 
as best estimator in agreement with the Bayesian framework. As a further remark, let us stress that the best fit parameters $\mathbf{p}_{\mathrm{BF}}$ may also significantly differ from the maximum likelihood values $\mathbf{p}_{\mathrm{ML}}$ because of parameter degeneracies. Actually, in order to have $\mathbf{p}_{\mathrm{BF}}=\mathbf{p}_{\mathrm{ML}}, \mathcal{L}(\mathbf{p})$ should be written as the product of the marginalized likelihood functions. Moreover, these latter should be Gaussian so that they are centred on the maximum likelihood values (therefore coincident with the median values). Actually, both these conditions are rarely met so that having $\mathbf{p}_{\mathrm{BF}} \neq \mathbf{p}_{\mathrm{ML}}$ is quite common.

The Markov Chains may also be used to extract constraints on some interesting derived quantities. To this aim, let us consider a generic function $y=g(\mathbf{p})$. Evaluating $y$ along the chains, we can build up the histogram of its values and then use the median and quantiles of this latter to infer the Bayesian confidence levels on $y$. Such a procedure will be used for the scaling model parameters $\log \eta_{0}$ and $\Upsilon_{\text {eff }}$ and for the projected and spherical DM mass fractions for each lens in the sample.

\subsubsection{The lensing merit function}

Dealing with the Einstein angle as a constraint is not straightforward. Indeed, for a given set of model parameters, $\xi_{\mathrm{E}}$ should be find solving Eq. (45) so that a lot of computing time should be spent to explore the four dimensional parameter space. Moreover, the photometric parameters $\left(n, I_{\mathrm{e}}, R_{\mathrm{eff}}\right)$ entering Eq. (45) are affected by their own uncertainties so that one should solve this relation many times by, for instance, bootstrapping the values of these quantities and looking for the corresponding uncertainty induced on the predicted $\xi_{\mathrm{E}}$. Fortunately, we can skip all these complications by resorting to the projected mass within the Einstein radius whose theoretical and observed values may be estimated from Eqs. (33) and (46).

Because of the measurement errors on $I_{\mathrm{e}}$ and $R_{\mathrm{eff}}$ and the uncertainty on $\Upsilon_{\text {eff }}$ coming from the one on $\Upsilon_{*}$, the predicted $M_{\text {proj }}^{\text {th }}\left(\xi_{\mathrm{E}}\right)$ is affected by an uncertainty which we naively quantity using error propagation as:

$\frac{\delta M_{\mathrm{proj}}^{\mathrm{th}}\left(\xi_{\mathrm{E}}\right)}{M_{\mathrm{proj}}^{\mathrm{th}}\left(\xi_{\mathrm{E}}\right)}=\sqrt{\left(\frac{\delta L_{\mathrm{T}}}{L_{\mathrm{T}}}\right)^{2}+\left(\frac{\delta \Upsilon_{\mathrm{eff}}}{\Upsilon_{\mathrm{eff}}}\right)^{2}}$

with:

$\frac{\delta L_{\mathrm{T}}}{L_{\mathrm{T}}}=\ln 10 \sqrt{\left(2 \delta \log R_{\mathrm{eff}}\right)^{2}+\left(\delta \log I_{\mathrm{e}}\right)^{2}}$,

$\frac{\delta \Upsilon_{\mathrm{eff}}}{\Upsilon_{\mathrm{eff}}}=\sqrt{\left(\frac{\delta L_{\mathrm{T}}}{L_{\mathrm{T}}}\right)^{2}+\left(\frac{\delta M_{\mathrm{vir}}}{M_{\mathrm{vir}}}\right)^{2}}$,

$\frac{\delta M_{\mathrm{vir}}}{M_{\mathrm{vir}}}=\sqrt{\left(\frac{\delta \Upsilon_{*}}{\Upsilon_{*}}\right)^{2}+\left(\frac{\delta L_{\mathrm{T}}}{L_{\mathrm{T}}}\right)^{2}}$,

note that we have here neglected the contribution of the error on $R_{\mathrm{E}}$ entering through the integral in Eq. (33). To take this into account, we should compute the numerical derivative $\mathrm{d} M_{\text {proj }}^{\text {th }}(\xi) / \mathrm{d} \xi$ for each choice of the model parameters. Such a computationally expensive procedure may be however skipped since, being $R_{\mathrm{E}}$ measured with a quite low error, the integral function may be well approximated as a constant over such a narrow range. As a consequence, the corresponding error term is safely negligible with respect to the ones due to the measurement uncertainties on $I_{\mathrm{e}}$ and $R_{\text {eff }}$. We stress, however, that neglecting this term makes our analysis more conservative since we are actually assuming that the final uncertainty on $M_{\text {proj }}^{\text {th }}\left(\xi_{\mathrm{E}}\right)$ is lower than its true value. The error on $R_{\mathrm{E}}$ is instead taken into account to compute the one on the observed projected mass being:

$\delta M_{\text {proj }}^{\mathrm{obs}}\left(\xi_{\mathrm{E}}\right) / M_{\text {proj }}^{\mathrm{obs}}\left(\xi_{\mathrm{E}}\right)=2 \delta R_{\mathrm{E}} / R_{\mathrm{E}}$

We finally define the lensing merit function as:

$\chi_{\text {lens }}^{2}(\mathbf{p})=\sum_{i=1}^{\mathcal{N}}\left[\frac{M_{\mathrm{proj}, i}^{\mathrm{obs}}\left(\xi_{\mathrm{E}}\right)-M_{\mathrm{proj}, i}^{\mathrm{th}}\left(\xi_{\mathrm{E}}, \mathbf{p}\right)}{\varepsilon_{\text {lens }, i}}\right]^{2}$

where the total uncertainty $\varepsilon_{\text {lens }, i}$ is given by the sum in quadrature of the theoretical and observational uncertainties estimated from Eqs. (51) and (52) and the sum is over the $\mathcal{N}$ lenses in the sample.

\subsubsection{The dynamics figure of merit}

Denoting with $\sigma_{\text {ap,obs }}$ the observed value, we define the following figure of merit for the dynamical data:

$\chi_{\mathrm{dyn}}^{2}(\mathbf{p})=\sum_{i=1}^{\mathcal{N}}\left[\frac{\sigma_{\mathrm{ap}, \mathrm{obs}}^{(i)}-\sigma_{\mathrm{ap}, \mathrm{th}}^{(i)}(\mathbf{p})}{\varepsilon_{\mathrm{dyn}, i}}\right]^{2}$

where $\varepsilon_{\text {dyn }}^{(i)}$ is obtained by summing in quadrature the theoretical and measurement uncertainties, while the sum is, as usual, over the $\mathcal{N}$ objects in the sample. Note that, although $R_{\text {ap }}$ is the same for all galaxies, the different measurements of $\sigma_{\text {ap }}$ actually probe a range of scaled radii $\xi_{\text {ap }}$ since the effective radius changes on a case-by-case basis. As a final remark, it is worth stressing that $\sigma_{\text {ap,th }} \propto \Upsilon_{\text {eff }}^{1 / 2}$, while $M_{\text {proj }}^{\text {th }}\left(\xi_{\mathrm{E}}\right) \propto \Upsilon_{\text {eff }}$. It is this diverse scaling with $\Upsilon_{\text {eff }}$ (and the other model parameters too) which makes combining lensing and dynamical data an efficient tool to partially break the degeneracies among model parameters thus strengthening the constraints we can obtain.

\subsection{The data}

The sums in Eqs. (53) and (54) run over the $\mathcal{N}$ objects in the sample. It is therefore vital to specify what this sample is made of. We consider here the 21 lens ETGs reported in Gavazzi et al. (2007) whose main properties are summarized in Table 1 where columns are as follows: 1. name (without the prefix SDSS); 2. lens redshift; 3 . source redshift; 4 . absolute $V$ magnitude (incremented by $5 \log h$ ); 5 . effective radius (in $\operatorname{arcsec}$ ); 6 . Einstein radius (in $\operatorname{arcsec}$ ); 7. aperture velocity dispersion (in $\mathrm{km} \mathrm{s}^{-1}$ ); 8. ratio between the aperture and the effective radii; 9. estimated $V$-band stellar $M / L$ ratio with its error. Note that we do not have colours for lens $\mathrm{J} 223840.2+075456$ so that $\Upsilon_{*}$ cannot be evaluated and this object will be excluded by the analysis. These lenses have been observed in the framework of the SLACS survey which aims at confirming through ACS imaging candidate lenses spectroscopically identified within the SDSS catalog (see Bolton et al. 2004, 2006, 2008, for further details). The SDSS velocity dispersions have been measured within a circular aperture of fixed radius $R_{\text {ap }}=1.5 \operatorname{arcsec}$ and spans the range $196 \leq \sigma_{\text {ap }} \leq 333 \mathrm{~km} / \mathrm{s}$ with a mean square velocity $\left\langle\sigma_{\mathrm{ap}}^{2}\right\rangle^{1 / 2} \simeq 248 \mathrm{~km} \mathrm{~s}^{-1}$. The lens galaxies have a mean redshift $\left\langle z_{L}\right\rangle \simeq 0.22$, but it is worth noting that the redshift range covered $\left(0.063 \leq z_{L} \leq 0.513\right)$ probes almost one order of magnitude (even if quite sparsely). The measured surface brightness 
has been fitted by a de Vaucouleurs (1948) model so that $n$ will be fixed to 4 in the following. Typical uncertainties on $R_{\mathrm{eff}}$ and $I_{\mathrm{e}}$ are quite small so that, following Bolton et al. (2008), we will set $\left(\delta \log I_{\mathrm{e}}, \delta \log R_{\mathrm{eff}}\right)=(0.020,0.015)$ for all galaxies in the sample. Gavazzi et al. (2007) provides also absolute $V$ band magnitude corrected for filter transformation and Galactic extinction and $K$ and evolution corrected to a common redshift $z=0.2$. When fitting Einstein radius and velocity dispersions, however, we need the luminosity at the lens redshift. This can be easily estimated as (Gavazzi et al. 2007):

$\log L_{V}\left(z_{L}\right)=\log L_{V}(z=0.2)-0.40\left(z_{L}-0.2\right)$

with:

$\log L_{V}(z=0.2)=\operatorname{dex}\left(-\frac{M_{V}-M_{V, \odot}}{2.5}\right)$

having set $\operatorname{dex}(x)=10^{x}$ and denoted by $M_{V}$ and $M_{V, \odot}=4.83$ the absolute magnitudes of the lens at $z=0.2$ and of the Sun, respectively. We further estimate the effective intensity as (Graham \& Driver 2005):

$I_{\mathrm{e}}=\frac{\left\langle I_{\mathrm{e}}\right\rangle}{n \mathrm{e}^{b_{n}} b_{n}^{-2 n} \Gamma(2 n)}$

with the effective surface brightness given by:

$\left\langle I_{\mathrm{e}}\right\rangle=L_{V}\left(z_{L}\right) / 2 \pi R_{\mathrm{eff}}^{2}$.

While $\sigma_{\text {ap }}$ is directly measured from the lens spectrum, the Einstein radii are determined by a parameterized procedure. The lens potential is modeled as a singular isothermal ellipse, while a non parametric technique is employed to reconstruct the source surface brightness profile. The Einstein radii is then estimated so that the lensed source matches the observed arc-like features. Typical uncertainties in $R_{\mathrm{E}}$ are of order 0.05 arcsec with a small variation among the lenses. Considering the different $R_{\mathrm{E}}$ values, we naively set $\delta R_{\mathrm{E}} / R_{\mathrm{E}}=0.05$ for all the sample objects. Table 1 summarizes the relevant quantities for our analysis.

\section{Results}

The sample of 21 SLACS lenses represents the input dataset needed to constrain the four model parameters $\left(\alpha, \alpha+\beta, s_{\mathrm{E}}, \mu_{\mathrm{vir}}\right)$ through the Bayesian likelihood analysis described above. As a first test, motivated by the above discussion, we fit the model to the full sample without any binning in luminosity thus giving us a quite large set of constraints (namely, $2 \times 21=42$ observable quantities vs. 4 parameters). Nevertheless, some care is needed when examining the results of the Markov Chain analysis.

In order the results to be reliable, one should carefully check that the chains have reached convergence, that is to say that the chains have fully explored the regions of high likelihood. Should this be the case, one should see the points of the chains for each single parameter oscillate around an average value or, put another way, the histograms of the parameters be single peaked (eventually, with a Gaussian shape). In order to test the convergence of the chains we resort to the test described in Dunkley et al. (2005, see also Dunkley et al. 2009) which is based on the analysis of the chain power spectra. Before checking for convegence, however, we first cut out the initial $30 \%$ of the chain in order to avoid the burn in period. Moreover, to reduce spurious correlations among parameters induced by the nature of the Markov Chain process, we thin the chain by taking 1 point each 25 . We find that the convergence test is well passed for a
Table 2. Results for the fit to the full lens sample.

\begin{tabular}{ccccc}
\hline \hline Par & mean & median & $68 \%$ CL & $95 \%$ CL \\
\hline$\alpha$ & -0.26 & -0.25 & $(-0.36,-0.16)$ & $(-0.43,-0.06)$ \\
$\alpha+\beta$ & 0.55 & 0.54 & $(0.47,0.63)$ & $(0.40,0.71)$ \\
$s_{\mathrm{E}}$ & -2.17 & -2.17 & $(-2.24,-2.10)$ & $(-2.31,-2.03)$ \\
$\mu_{\mathrm{vir}}$ & 13.60 & 12.96 & $(9.52,17.81)$ & $(7.60,23.29)$ \\
\hline
\end{tabular}

chain containing $10^{5}$ points which reduces to 2801 after the burn in cut and the thinning. Such a large sample is more than sufficient to sample the four dimensional parameter space. Note that we have also tried to change the burn in cut and thinning thus checking that the results are unaffected by these (somewhat arbitrary) choices.

\subsection{Constraints on the model parameters}

The results obtained fitting our model to the full SLACS sample are summarized in Table 2 which reports, for each parameter, mean and median values and 68 and $95 \%$ confidence limits. First, we note that the best fit model parameters turns out to be:

$\left(\alpha, \alpha+\beta, s_{\mathrm{E}}, \mu_{\mathrm{vir}}\right)=(-0.34,0.52,-2.16,12.22)$

giving $\chi^{2} /$ dof $=63.34 / 38=1.66$. From a statistical point of view, such a reduced $\chi^{2}$ value could seem too large. Actually, a large $\chi^{2}$ /dof may be due to a model failure or to an intrinsic scatter which has not been taken into account. Such a second possibility is indeed the more realistic considering that, having stacked all the lens together notwithstanding their different photometric and mass properties, a certain degree of scatter is indeed expected. Moreover, the overall quality of the fit appears to be quite good. In order to quantify the agreement between the data and the model for the best fit parameters, we first introduce the two following quantities:

$$
\begin{aligned}
& \frac{\Delta M_{\mathrm{E}}}{\varepsilon_{\text {lens }}}=\frac{M_{\mathrm{proj}}^{\mathrm{obs}}\left(\xi_{\mathrm{E}}\right)-M_{\mathrm{proj}}^{\mathrm{th}}\left(\xi_{\mathrm{E}}, \mathbf{p}_{\mathrm{bf}}\right)}{\varepsilon_{\text {lens }}} \\
& \frac{\Delta \sigma_{\mathrm{dap}}}{\varepsilon_{\mathrm{dyn}}}=\frac{\sigma_{\mathrm{ap}, \mathrm{obs}}-\sigma_{\mathrm{ap}, \mathrm{th}}\left(\mathbf{p}_{\mathrm{bf}}\right)}{\varepsilon_{\mathrm{dyn}}}
\end{aligned}
$$

with $\mathbf{p}_{\mathrm{bf}}$ the set of best fit parameters. It turns out that:

$$
\begin{aligned}
& \left\langle\Delta M_{\mathrm{E}} / \varepsilon_{\text {lens }}\right\rangle=-0.12, \operatorname{rms}\left(\Delta M_{\mathrm{E}} / \varepsilon_{\mathrm{E}}\right)=1.09 \\
& \left\langle\Delta \sigma_{\mathrm{ap}} / \varepsilon_{\mathrm{dyn}}\right\rangle=-0.04, \operatorname{rms}\left(\Delta \sigma_{\mathrm{ap}} / \varepsilon_{\mathrm{dyn}}\right)=1.35
\end{aligned}
$$

The low values of $\left\langle\Delta M_{\mathrm{E}} / \varepsilon_{\text {lens }}\right\rangle$ and $\left\langle\Delta \sigma_{\mathrm{ap}} / \varepsilon_{\text {dyn }}\right\rangle$ are a clear evidence that the model is working well, while the larger rms values are expected considering that we are stacking galaxies with different intrinsic and environmental properties. It is worth stressing, moreover, that both the projected mass within the Einstein ring and the aperture velocity dispersion predicted by the best fit model is well within $1 \sigma$ from the observed values for most of the galaxies, while there is only one lens ${ }^{8}$ with a theoretically

\footnotetext{
8 This is the lens identified as SDSS J234111.6+000019. In all the fits we will consider, this is, indeed, the lens showing the larger normalized residuals for both the projected mass and the velocity dispersion so that it is possible that some peculiar feature is at work. However, in order to not reduce the number of constraints without a definite motivation, we prefer to retain this object in the sample even if we caution the reader againt overrating its large residuals.
} 
Table 3. Results for the fit to the binned lenses.

\begin{tabular}{cccccc}
\hline \hline Sample & $M_{V}$ & $\left(\alpha, \alpha+\beta, s_{\mathrm{E}}, \mu_{\mathrm{vir}}\right)_{b f}$ & $\Delta M_{\mathrm{E}} / \varepsilon_{\text {lens }}$ & $\Delta \sigma_{\mathrm{ap}} / \varepsilon_{\mathrm{dyn}}$ & $\chi^{2} / \mathrm{dof}$ \\
\hline B1 & $-22.40_{-0.55}^{+0.15}$ & $(-0.12,1.31,-2.19,72.42)$ & $(-0.12,0.58)$ & $(-0.11,0.57)$ & 0.55 \\
B2 & $-22.15_{-0.05}^{+0.09}$ & $(-0.45,1.22,-2.20,73.30)$ & $(-0.16,1.42)$ & $(-0.06,1.45)$ & 3.08 \\
B3 & $-21.83_{-0.19}^{+0.16}$ & $(-0.41,0.44,-2.20,8.51)$ & $(-0.03,0.55)$ & $(-0.12,1.11)$ & 1.15 \\
B4 & $-21.33_{-0.25}^{+0.39}$ & $(-0.44,0.33,-2.39,5.00)$ & $(-0.11,0.65)$ & $(-0.04,0.34)$ & 0.55 \\
\hline
\end{tabular}

predicted velocity dispersion $3 \sigma$ larger than the observed value. We therefore safely conclude that the model have successfully fitted the data for the lens sample we are considering.

Having established the validity of our model, let us now discuss the constraints on its parameters. As a first lesson, we note that models with $\alpha>0$ are definitely excluded contrary to the discussion in Sect. 2 where we have shown that negative $\alpha$ values lead to a global $M / L$ ratio diverging for $\eta \rightarrow 0$. However, such an unphysical behaviour is actually never approached since, as we have seen in Sect. 5, our phenomenological ansatz do not fit realistic models for $\eta \leq 0.01$ so that one has not to trust its extrapolation in the very inner regions. On the other hand, the analysis in Sect. 5 have shown that models with $\alpha \leq 0$ are needed to fit the global $M / L$ ratios of different dark halo models. It is therefore reassuring that the likelihood analysis indeed prefer this kind of models thus leading further support to our phenomenological approach. As a further remark, we also note that the values of $\alpha$ and $\alpha+\beta$ are typical of cored dark halo density profiles (such as the NIS and Burkert models). However, the very small $\alpha$ values obtained when considering the NFW model are also partly due to the adopted mass-concentration relation so that it is likely that changing the $c-M_{\text {vir }}$ relation may lead to larger and still negative $\alpha$ values. Exploring this issue is outside our aims here, but we argue that cored profiles are preferred on the basis of our phenomenological approach.

A further success of our model is represented by the constraints on $s_{\mathrm{E}}$, i.e. the logarithmic slope of the total density profile at the scaled Einstein radius, i.e. $s_{\mathrm{E}}=\mathrm{d} \log \rho / \mathrm{d} \log \eta(\eta=$ $\left.R_{\mathrm{E}} / R_{\mathrm{eff}}\right)$. Previous analysis in literature have typically fitted the observed Einstein radius and velocity dispersion using a parameterized density profile. For instance, Koopmans \& Treu (2003a,b) have fitted the data on 0047-281 and B1608+656 assuming $\rho \propto r^{-\gamma}$ and finding $\gamma=-1.90_{-0.23}^{+0.05}$ and $\gamma=-2.03 \pm 0.14$, respectively. Using the same methodology, but averaging over a sample of 15 SLACS lenses, Koopmans et al. (2006) have found again $\gamma=-2.01_{-0.03}^{+0.02}$. As yet said before and also stressed by the same authors, these may be considered as constraints on the logarithmic slope at the Einstein radius since this is the range mainly probed by the data. It is therefore reassuring that, using a similar data analysis but a radically different parametrization, we get compatible constraints on $s_{\mathrm{E}}$ thus strengthening our results.

Finally, we consider the $\mu_{\text {vir }}$ parameter. It is easy to transform the constraints on this quantity into one on the DM mass fraction at the virial radius thus getting:

$\left\langle f_{\text {vir }}\right\rangle=\left(f_{\text {vir }}\right)_{\text {med }}=0.92$,

$68 \%$ CL : $(0.89,0.94), 95 \% \mathrm{CL}:(0.87,0.96)$.

Using $f_{\text {vir }}=1-\varepsilon_{\mathrm{SF}} f_{b}$ with $f_{b} \simeq 0.17$, we get $\left\langle\varepsilon_{\mathrm{SF}}\right\rangle \simeq 0.47$, i.e. only $\sim 50 \%$ of the baryonic mass is converted into stars. This is consistent with semianalytic galaxy formation models (Benson et al. 2000) which typically use values in the range $(0.2,1.0)$.
From the observational point of view, the situation is much more complicated. On the one hand, studies matching the luminosity and mass functions (2002; Guzik \& Seljak 2002) or $M / L$ gradients (2005) of local galaxies suggest $\varepsilon_{\mathrm{SF}} \sim 0.25$, while stellar masses in the SDSS survey (Padmanabhan et al. 2004) imply $\varepsilon_{S F} \simeq 0.2-0.8$. Given such weak observational constraints, we can only argue that our estimate of $\mu_{\mathrm{vir}}$ and hence $\varepsilon_{\mathrm{SF}}$ is fully realistic. It is worth noting, however, that both observations and theoretical modelling point towards a U-shaped trend of $\varepsilon_{\mathrm{SF}}$ with the mass (Benson et al. 2000; 2005; Conroy \& Wechsler 2008). One could therefore argue that our approach based on a universal $\varepsilon_{\mathrm{SF}}$ is unmotivated, but the small mass range probed by the lenses and the good results obtained let us conclude that this choice does not bias anyway the constraints on the quantities of interest.

\subsection{Binning the lenses}

A simple analysis of the best fit residual to the full sample shows that there is actually no correlation with either the lens redshift, the total luminosity and stellar mass. Such a result may argue in favour of our assumption about the universality of the parameters $\left(\alpha, \alpha+\beta, s_{\mathrm{E}}, \mu_{\mathrm{vir}}\right)$. However, this can also be a consequence of an erroneous estimation of the errors or of insufficient statistics. To further explore this issue, we therefore divide the lenses in four almost equally populated bins (denoted as B1, B2, B3, B4) according to their absolute $V$ magnitude and run our MCMC algorithm using chains with $2 \times 10^{5}$ points reducing to 5601 after burn in cut and thinning. Note that, because we use now a smaller number of constraints (namely, 6-8 vs. 38), we have to run longer chains in order to achieve the same convergence as with the full sample. We summarise in Table 3 the best fit values and statistics of residuals for the fits to the binned samples, reporting in the second column the median value and the full range of $M_{V}$ in each bin, while the two numbers in columns 4 and 5 refer to the mean and rms values of the lensing and dynamics residuals, respectively. Table 4 reports the constraints on the model parameters after marginalization using the notation $x_{-y_{1}-y_{2}}^{+z_{1}+z_{2}}$ to mean that $x$ is the median value, while $68 \%$ and $95 \%$ CL read $\left(x-y_{1}, x+z_{1}\right)$ and $\left(x-y_{2}, x+z_{2}\right)$, respectively. These results allow us to make some interesting considerations.

As a first remark, we note that the fit is still successful as witnessed by both the low normalized residuals and reduced $\chi^{2}$ values ${ }^{9}$. Actually, one could wonder why the reduced $\chi^{2}$ values for bin B1 and B4 are smaller than unity thus arguing for a possible overestimate of the errors. While this is possible since our estimate is based on a naive propagation not taking into account correlations among the uncertainties on photometric quantities, we nevertheless note that the $\chi^{2} /$ dof values for bins B2 and B3 do not present such a problem. Notwithstanding the solution of this

\footnotetext{
9 The high $\chi^{2} /$ dof for $\mathrm{B} 2$ is due to to the peculiar lens SDSS $\mathrm{J} 234111.6+000019$ so remember the caveat yet quoted before.
} 
Table 4. Marginalized constraints on the model parameters for different bins.

\begin{tabular}{|c|c|c|c|c|}
\hline Sample & $\alpha$ & $\alpha+\beta$ & $s_{\mathrm{E}}$ & $\mu_{\mathrm{vir}}$ \\
\hline B1 & $-0.11_{-0.14}^{+0.16}+0.30$ & $0.86_{-0.24}^{+0.29}{ }_{-0.41}^{0.58}$ & $-2.18_{-0.11-0.23}^{+0.13}$ & $27.01_{-12.62}^{+23.32}+43.23$ \\
\hline B2 & $-0.29_{-0.11-0.15}^{+0.14+0.23}$ & $0.91_{-0.21}^{+0.20}{ }_{-0.42}^{+0.42}$ & $-2.12_{-0.14-0.28}^{+0.14+0.27}$ & $41.20_{-20.87-32.06}^{+22.44}+32.07$ \\
\hline B3 & $-0.28_{-0.11}^{+0.10+0.23}$ & $0.54_{-0.12}^{+0.15}{ }_{-0.21}^{+0.31}$ & $-2.17_{-0.11-0.19}^{+0.11+0.21}$ & $12.02_{-4.44}^{+8.06}{ }_{-6.62}^{22.07}$ \\
\hline B4 & $-0.23_{-0.12}^{+0.14}{ }_{-0.20}^{0.19}$ & $0.51_{-0.12}^{+0.22}{ }_{-0.20}^{+0.58}$ & $-2.34_{-0.13}^{+0.12+0.26}-0.27$ & $8.95_{-2.88}^{+6.29}+21.25$ \\
\hline
\end{tabular}
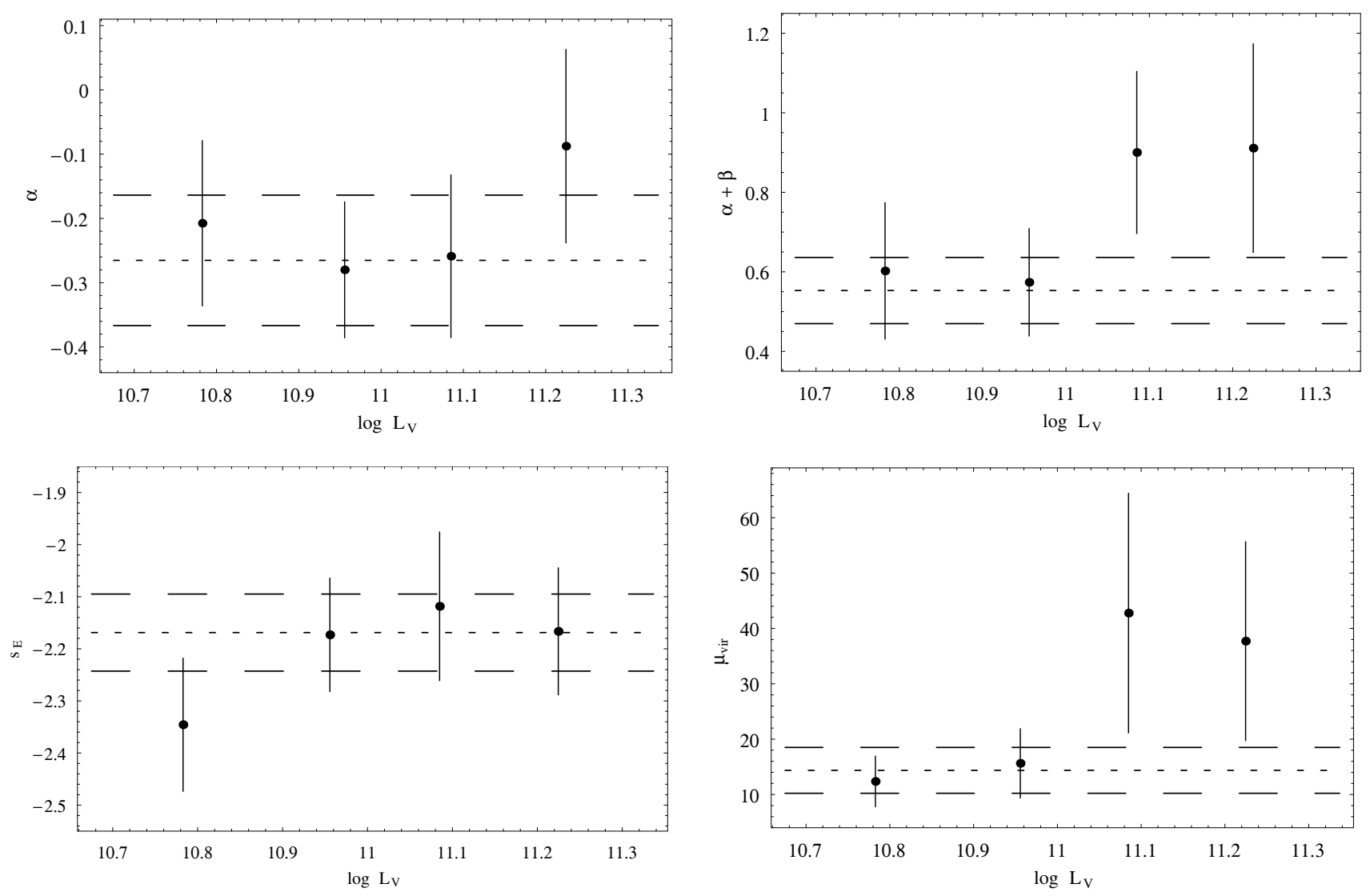

Fig. 1. Variation of the model parameters with the median $\log L_{V}$ of each bin. The central dot denotes the median values, while the vertical bars span the $68 \%$ CL range. We overplot the constraints from the fit to the full sample as horizontal lines with the short dashed one referring to the median value and the long dashed ones delimiting the $68 \%$ CL range.

ambiguity, we however stress that the constraints on the model parameters are not biased.

It is interesting to compare the results on the marginalized model parameters with the corresponding ones for the full sample. To this aim, we look at Fig. 1 where we have plotted the median and $68 \%$ CL estimate of each parameter as a function of the median $\log L_{V}$ of the bin and overplotted the results from the fit to the full sample. As it is clear, it is not possible to infer any statistically reliable trend for $\alpha$ which is indeed constant within the errors and compatible with the result from the fit to all lenses. A similar discussion may also be done for $\alpha+\beta, \mu_{\text {vir }}$ and $s_{\mathrm{E}}$, which due to large uncertainties cannot allow to determine strong trends with luminosity. However, these figures seem to suggest interesting trends as a function of $\log L_{V}$, which we will better explore in a future paper enlarging the sample. Our results give issues about a dependence of the parameters above on luminosity and stellar mass, with brighter galaxies having on average higher values for $\alpha+\beta$ and $\mu_{\mathrm{vir}}$ and a profile more similar to the isothermal sphere. Finally, an increase of $\mu_{\text {vir }}$ with luminosity and stellar mass implies that the star formation efficiency $\varepsilon_{\mathrm{SF}}$ decreases with these quantities. Indeed, this is consistent with the observational and theoretical studies (Benson et al. 2000; Napolitano et al. 2005; Conroy \& Wechsler 2008; Tortora et al. 2009a; Napolitano et al. 2009) showing that more massive galaxies are less able to convert gas into stars.

As a general remark, it is worth noting that all the eventual trends seen in Fig. 1 become statistically meaningless should we have plotted the 95 instead of the $68 \%$ CL range. In contrast, one could equally well conclude that, to a first reasonable approximation, the model parameters $\left(\alpha, \alpha+\beta, s_{\mathrm{E}}, \mu_{\mathrm{vir}}\right)$ do not depend on the luminosity. As we will show later, the derived quantities (such as the DM mass fractions and the scalelength parameter $\left.\log \eta_{0}\right)$ are consistent within the bins and the full sample. Motivated by these considerations, we therefore hereafter refer 
Table 5. Median values and 68 and 95\% CL ranges for the DM mass fractions using the chain for the fit to the full lens sample.

\begin{tabular}{|c|c|c|c|c|}
\hline Name & $f_{\text {proj }}\left(R_{\mathrm{E}}\right)$ & $f_{\mathrm{DM}}\left(R_{\mathrm{E}}\right)$ & $f_{\mathrm{DM}}\left(R_{\mathrm{eff}}\right)$ & $\nabla_{\uparrow} \Upsilon$ \\
\hline J002907.8-005550 & $0.615_{-0.016}^{+0.016+0.031}$ & $0.564_{-0.016}^{+0.016+0.031}$ & $0.602_{-0.019+0.041}^{+0.018}$ & $0.47_{-0.12-0.22}^{+0.13+0.29}$ \\
\hline J015758.9-005626 & $0.660_{-0.019}^{+0.018+0.041}$ & $0.601_{-0.016}^{+0.014}+0.027$ & $0.623_{-0.017-0.036}^{+0.015+0.029}$ & $0.56_{-0.14-0.26}^{+0.15}$ \\
\hline J021652.5-081345 & $0.715_{-0.013}^{+0.012}{ }_{-0.026}^{+0.024}$ & $0.687_{-0.011}^{+0.010}+0.020$ & $0.714_{-0.016}^{+0.016+0.035}$ & $0.62_{-0.19-0.32}^{+0.20+0.42}$ \\
\hline J025245.2+00358 & $0.666_{-0.016}^{+0.016}{ }_{-0.034}^{+0.028}$ & $0.621_{-0.013}^{+0.011}+0.026$ & $0.651_{-0.016}^{+0.017}+0.035$ & $0.55_{-0.15-0.26}^{+0.16+0.34}$ \\
\hline J033012.1-002052 & $0.631_{-0.021}^{+0.020}+0.037$ & $0.558_{-0.018}^{+0.017}+0.036$ & $0.570_{-0.018}^{+0.017}+0.037$ & $0.53_{-0.13-0.23}^{+0.15}+0.29$ \\
\hline $\mathrm{J} 072805.0+383526$ & $0.563_{-0.021}^{+0.019}{ }_{-0.043}^{+0.036}$ & $0.474_{-0.018}^{+0.024}+0.036$ & $0.482_{-0.017}^{+0.016}+0.035$ & $0.46_{-0.11}^{+0.11}+0.20$ \\
\hline J080858.8+470639 & $0.609_{-0.019}^{+0.017-0.031}$ & $0.543_{-0.016}^{+0.013}{ }_{-0.031}^{+0.027}$ & $0.570_{-0.017}^{+0.016}+0.037$ & $0.48_{-0.12-0.22}^{+0.13+0.27}$ \\
\hline J090315.2+411609 & $0.678_{-0.023}^{+0.021+0.038}$ & $0.615_{-0.019+0.039}^{+0.019+0.035}$ & $0.627_{-0.019}^{+0.019+0.039}$ & $0.61_{-0.15-0.28}^{+0.16+0.65}$ \\
\hline J091205.3+002901 & $0.730_{-0.012}^{+0.015}{ }_{-0.025}^{+0.032}$ & $0.714_{-0.015-0.031}^{+0.018}$ & $0.732_{-0.017-0.034}^{+0.019}$ & $0.61_{-0.20}^{+0.22}+0.45$ \\
\hline J095944.1+041017 & $0.575_{-0.021}^{+0.019}{ }_{-0.047}^{+0.036}$ & $0.523_{-0.026}^{+0.023+0.045}$ & $0.566_{-0.025}^{+0.022+0.041}$ & $0.42_{-0.11-0.20}^{+0.12}$ \\
\hline $\mathrm{J} 102332.3+42300$ & $0.546_{-0.020}^{+0.020}+0.037$ & $0.454_{-0.018}^{+0.018}+0.034$ & $0.465_{-0.017}^{+0.017+0.038}$ & $0.44_{-0.11}^{+0.11+0.19}$ \\
\hline $\mathrm{J} 110308.2+532228$ & $0.705_{-0.017}^{+0.023+0.050}+0.035$ & $0.692_{-0.023}^{+0.028}+0.056$ & $0.707_{-0.019}^{+0.022}+0.048$ & $0.54_{-0.18-0.33}^{+0.19+0.39}$ \\
\hline J120540.4+491029 & $0.622_{-0.016}^{+0.016}{ }_{-0.033}^{+0.029}$ & $0.573_{-0.016}^{+0.015}{ }_{-0.032}^{+0.030}$ & $0.610_{-0.018}^{+0.018+0.043}$ & $0.48_{-0.13-0.23}^{+0.13+0.29}$ \\
\hline $\mathrm{J} 125028.3+052349$ & $0.592_{-0.018}^{+0.018}{ }_{-0.039}^{+0.033}$ & $0.527_{-0.017}^{+0.015}{ }_{-0.034}^{+0.031}$ & $0.559_{-0.018}^{+0.016}{ }_{-0.040}^{+0.034}$ & $0.46_{-0.11}^{+0.12}+0.25$ \\
\hline $\mathrm{J} 140228.1+632133$ & $0.654_{-0.014-0.035}^{+0.016}$ & $0.605_{-0.013}^{+0.012+0.023}$ & $0.636_{-0.017}^{+0.016+0.036}$ & $0.53_{-0.14-0.25}^{+0.15}$ \\
\hline J142015.9+601915 & $0.489_{-0.043}^{+0.033}{ }_{-0.088}^{+0.063}$ & $0.437_{-0.056-0.103}^{+0.045+0.081}$ & $0.488_{-0.045-0.099}^{+0.034+0.064}$ & $0.34_{-0.09-0.16}^{+0.09+0.19}$ \\
\hline $\mathrm{J} 162746.5-00535$ & $0.666_{-0.015}^{+0.013}{ }_{-0.030}^{+0.025}$ & $0.626_{-0.013+0.012+0.023}^{+0.025}$ & $0.659_{-0.017-0.035}^{+0.017}$ & $0.54_{-0.16-0.26}^{+0.16+0.34}$ \\
\hline $\mathrm{J} 163028.2+452036$ & $0.639_{-0.022}^{+0.020}{ }_{-0.045}^{+0.036}$ & $0.568_{-0.018}^{+0.017+0.032}$ & $0.579_{-0.018}^{+0.017}{ }_{-0.037}^{+0.031}$ & $0.55_{-0.14-0.25}^{+0.14+0.29}$ \\
\hline $\mathrm{J} 230053.2+002238$ & $0.660_{-0.016}^{+0.015}{ }_{-0.033}^{+0.028}$ & $0.615_{-0.014}^{+0.011+0.025}$ & $0.647_{-0.017}^{+0.016}+0.036$ & $0.54_{-0.15}^{+0.15}+0.233$ \\
\hline $\mathrm{J} 230321.7+14221$ & $0.646_{-0.015-0.032}^{+0.013}$ & $0.608_{-0.016}^{+0.015}{ }_{-0.032}^{+0.030}$ & $0.643_{-0.019}^{+0.018+0.038}+0.033$ & $0.50_{-0.14-0.25}^{+0.15}$ \\
\hline $\mathrm{J} 234111.6+000019$ & $0.659_{-0.014-0.030}^{+0.017}+0.025$ & $\begin{array}{r}0.627_{-0.016-0.033}^{+0.014}+0.030 \\
\end{array}$ & $\begin{array}{l}0.659_{-0.019}^{+0.017}+0.034 \\
\end{array}$ & $0.51_{-0.15-0.26}^{+0.16+0.33}$ \\
\hline
\end{tabular}

to the constraints from the fit to the full lens sample. We, however, plan to further explore this issue with a larger lens sample covering a wider $M_{V}$ range in order to both improve the statistics and increase the number of points to infer any possible trend with the luminosity.

\subsection{The DM mass fraction}

Although the model we are testing has been introduced as an effective one to describe the galaxy, it is nevertheless an ideal tool to derive model independent constraints on the DM content of ETGs. Indeed, Eq. (48) shows that constraining $\Upsilon(r)$ is the same as constraining the spherical DM mass fraction $f_{\mathrm{DM}}(r)$. In order to quantify these results, we summarize in Table 5 , the median and 68 and $95 \%$ CL ranges for the projected mass fraction $f_{\text {proj }}\left(R_{\mathrm{E}}\right)$ at the Einstein radius and for the spherial DM mass fraction at both the Einstein and effective radii. To this end, we compute these quantities for each point of the chain obtained from the fit to the full lens sample after burn in cut and thinning.

Before discussing the constraints we obtain, it is worth wondering whether they depend on the choice to fit the full lens sample or the binned subsamples. To this end, we plot in Fig. 2 the median values (with their 68\% CL ranges) for the quantities of interest. It is worth noting that the errors bars are actually underestimated since they do not take into account the uncertainties on the stellar $M / L$ ratio and the photometric parameters $\left(I_{\mathrm{e}}, R_{\mathrm{eff}}\right)$. Indeed, the typical uncertainty increases in this case up to $\sim 0.1$ so that fully dominates the error induced by the model parameters. We have, however, adopted this choice in order to improve the reliability of the figures. With this caveat in mind, Fig. 2 shows that there is essentially a quite good agreement between the two estimates. A direct fit $^{10}$ (without taking errors into account) indeed gives:

$f_{\text {proj }}^{\mathrm{Bin}}\left(R_{\mathrm{E}}\right)=0.04+0.93 f_{\text {proj }}^{\mathrm{All}}\left(R_{\mathrm{E}}\right)\left(\sigma_{\mathrm{rms}}=0.02\right)$,

$f_{\mathrm{DM}}^{\mathrm{Bin}}\left(R_{\mathrm{E}}\right)=0.08+0.86 f_{\mathrm{DM}}^{\mathrm{All}}\left(R_{\mathrm{E}}\right)\left(\sigma_{\mathrm{rms}}=0.04\right)$,

$f_{\mathrm{DM}}^{\mathrm{Bin}}\left(R_{\mathrm{eff}}\right)=-0.003+0.99 f_{\mathrm{DM}}^{\mathrm{All}}\left(R_{\mathrm{eff}}\right)\left(\sigma_{\mathrm{rms}}=0.03\right)$.

Considering that the typical uncertainties are much larger than the offsets and that the slopes of these relations are close to 1 , we hereafter only considers the results from the fit to the full

${ }_{10}$ Both in Fig. 2 and in the fits presented in this section, we first correct for the small asymmetry in the errors following the prescriptions given in D' Agostini (2004). Denoting with $x$ the central value and with $\Delta_{ \pm}$ the negative and positive error so that the $68 \%$ CL reads $\left(x-\Delta_{-}, x+\Delta_{+}\right)$, the corrected value is $x_{\text {corr }}=x+\left(\Delta_{+}-\Delta_{-}\right)$with a symmetric uncertainty $\Delta_{\text {corr }}=\left(\Delta_{+}+\Delta_{-}\right) / 2$. Note that, since the $68 \%$ CL ranges in Table 5 are indeed quite symmetric, these corrections are actually negligible for most of the lenses in our sample. 

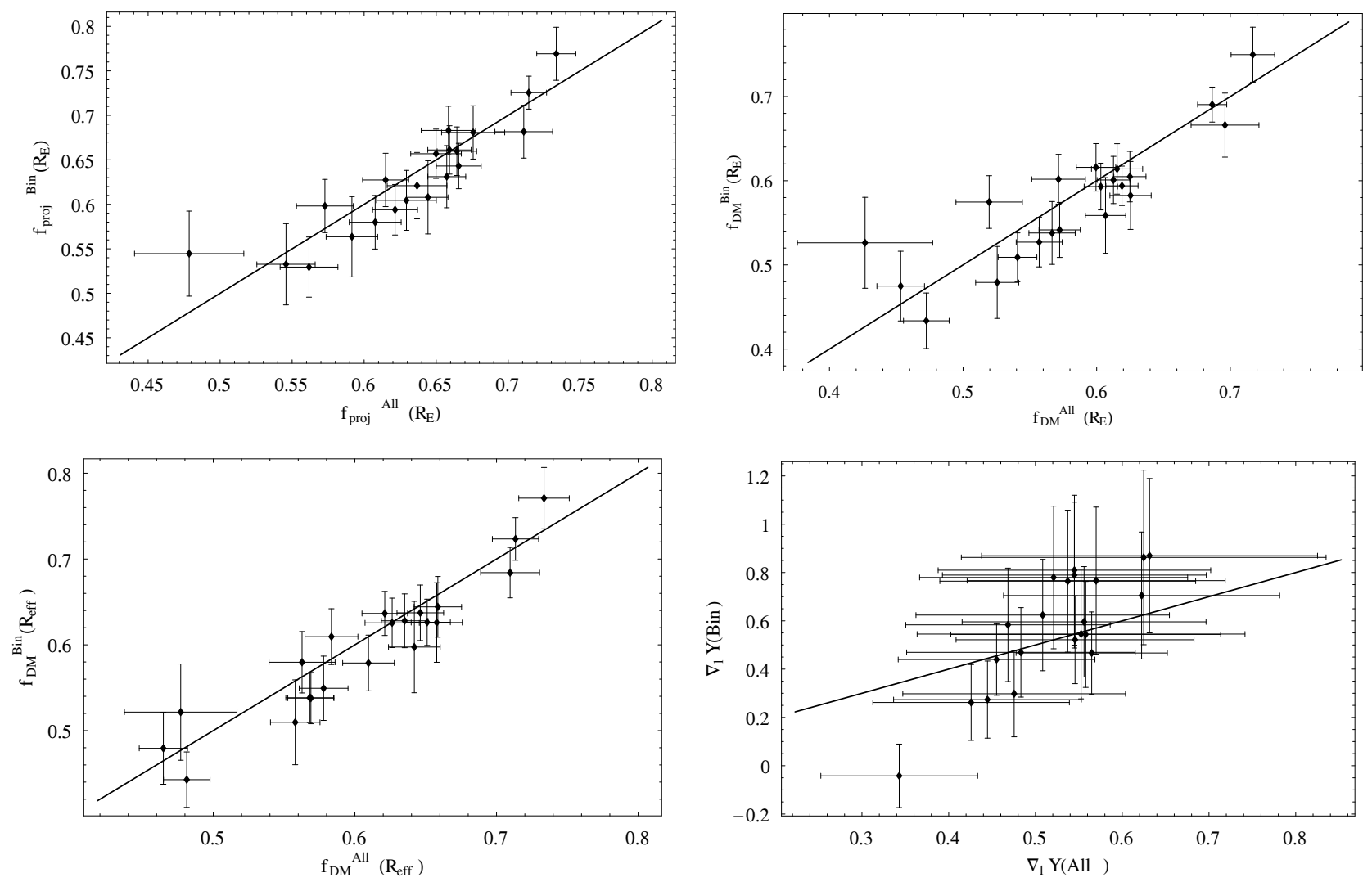

Fig. 2. Comparison between different DM content estimators as constrained from the fit to the full or binned samples. Error bars refer to the $68 \%$ CL ranges. The solid line superimposed on the data is the diagonal $f(x)=x$.

sample as our estimates of the DM mass fractions ${ }^{11}$ summarized in Table 5.

We can further explore the viability of our results comparing our estimated $f_{\mathrm{DM}}\left(R_{\mathrm{eff}}\right)$ with previous estimates in literature. Actually, a detailed comparison is not possible because our systems are at an intermediate redshift $(z \simeq 0.1-0.5)$, while dynamical and X-ray studies typically refer to nearby galaxies. Moreover, such estimates are model dependent relying on a given parametrization of the dark halo, while our constraints come from a phenomenological and hence model independent analysis. Since it is not possible to take quantitatively into account these differences, we caution the reader to not overrate any agreement or disagreement among our own and literature results.

As a first preliminary step, we briefly review some of the previous determinations. An accurate quantitative estimate of $f_{\mathrm{DM}}\left(R_{\mathrm{eff}}\right)$ has been obtained by Gerhard et al. (2001) from a dynamical analysis of the line profiles of 21 mostly luminous, slowly rotating and nearly round ETGs with well measured velocity dispersion and circular velocity profiles. According to their preferred minimum halo models, the DM mass fraction is $f_{\mathrm{DM}}\left(R_{\text {eff }}\right) \simeq 10-40 \%$ at the effective radius, finding that their central dynamical $M / L$ well agree with stellar $M / L$ estimated from synthetic models assuming a Salpeter IMF. Using orbit superposition method, Thomas et al. (2005) managed to reproduce in detail the observed kinematics of NGC 4807 finding $f_{\mathrm{DM}}\left(R_{\mathrm{eff}}\right)=0.21 \pm 0.14$ taking for $\Upsilon_{*}$ a guess from populations synthesis models with a Kroupa IMF. A larger sample of

\footnotetext{
11 The complete set of constraints, including the ones from the fit to the binned samples, are available on request to the authors.
}

24 ETGs with dynamics within $\sim 1-2 R_{\text {eff }}$ reconstructed from integral field spectroscopy was considered by Cappellari et al. (2006). Both two integrals analytic models and three integral orbit superposition agree each other giving for $f_{\mathrm{e}}$ a median value $\simeq 0.3$ using a Kroupa IMF as input to the estimate of $\Upsilon_{*}$. Analyzing a sample of local galaxies, Tortora et al. (2009a) found that luminous galaxies have typical values of $f_{\mathrm{DM}}\left(R_{\mathrm{eff}}\right) \sim$ $0.3-0.4$, using a Salpeter IMF, corresponding to $\sim 0.6-0.7$ for a Chabrier IMF. Finally, stacking the same 22 lenses we are using, Gavazzi et al. (2007) used a combined weak and strong lensing analysis leaving the stellar $M / L$ as a free parameter of the fit, finding out $f_{\mathrm{DM}}\left(R_{\mathrm{eff}}\right)=0.27 \pm 0.04$. These authors estimate an average value for stellar $M / L$ of $\sim 3.1$ (using $h=0.7$ ), which is 1.5 times the median value we obtain using a Chabrier IMF. Renormalizing all the estimates to the Chabrier IMF, the estimates for $f_{\mathrm{DM}}\left(R_{\text {eff }}\right)$ then span the range $\sim(0.30,0.70)$. Looking at Table 5, one can see that the values for $f_{\mathrm{DM}}\left(R_{\mathrm{eff}}\right)$ span a narrow range with $f_{\mathrm{DM}}\left(R_{\mathrm{eff}}\right) \simeq 0.61 \pm 0.07$ averaging over the 21 lenses in the sample. Such a value is in agreement with local estimates even if it is worth noting that most of the lenses have values of $f_{\mathrm{DM}}\left(R_{\text {eff }}\right)$ at the upper end of the local range. Indeed, our estimates are on average consistent with the local ones obtaibed in Tortora et al. (2009a) when using the same Chabrier IMF adopted here. A more careful and homogeneous (with respect to the local galaxies) sample is needed to analyze the possible evolution of DM fraction with redshift which is not the case here.

It has been recently reported in literature (Benson et al. 2000; Marinoni \& Hudson 2002; Graham \& Guzman 2003; van den Bosch et al. 2003a,b; Napolitano et al. 2005; Tortora et al. 2009a,b) that the DM content correlates with the total 

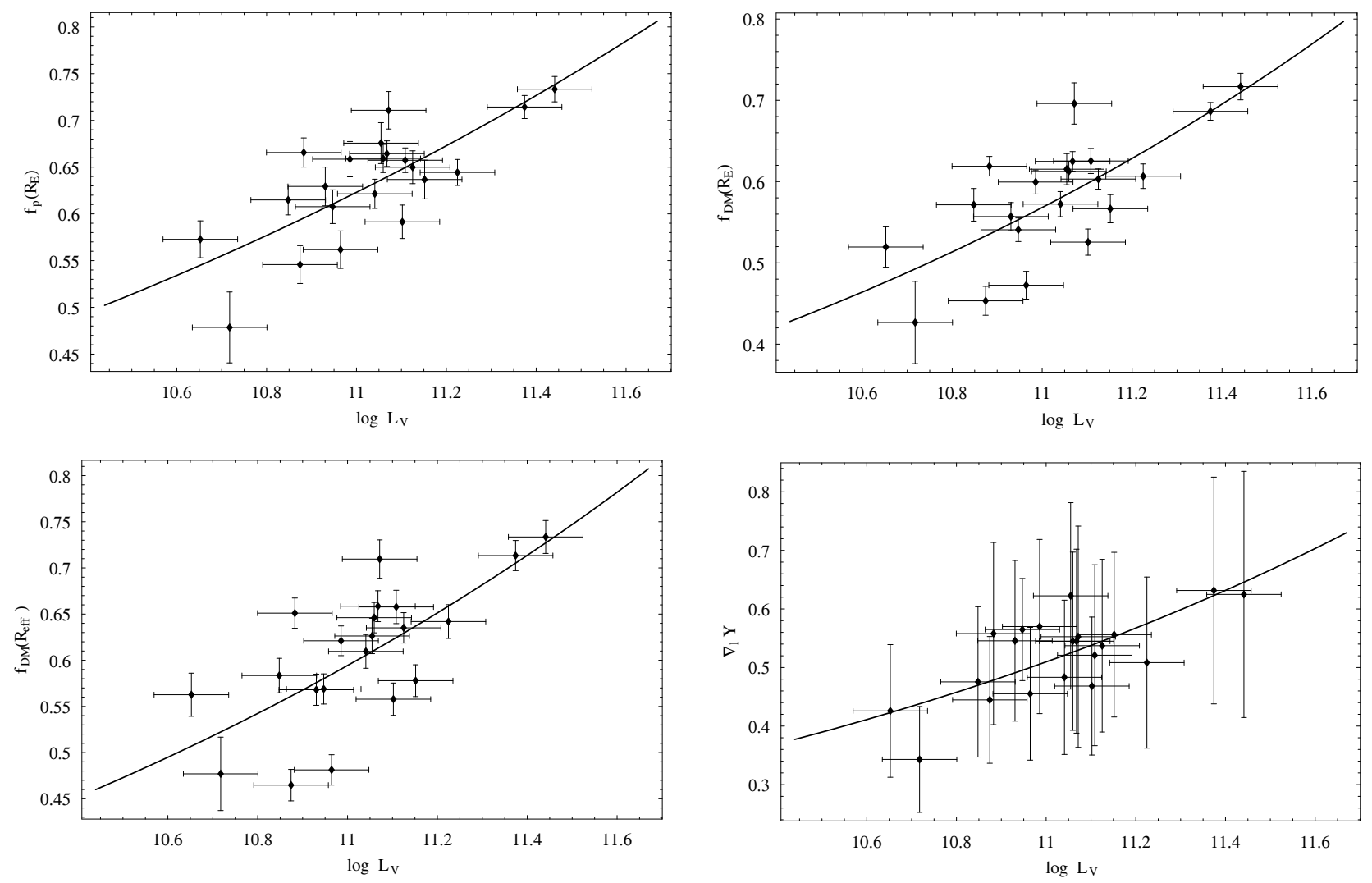

Fig. 3. DM mass fractions and empirical $M / L$ gradient vs total luminosity $\log L_{V}$. Errors bars refer to the $68 \% \mathrm{CL}$ ranges, while the superimposed solid lines refer to the best fit relations discussed in the text.

luminosity, with more luminous (and massive galaxies) having higher values of $f_{\mathrm{DM}}\left(R_{\mathrm{eff}}\right)$. This is what we indeed observe in Fig. 3 where the projected and spherical DM mass fractions (at both the Einstein and effective radii) are found to correlate with $\log L_{V}$, with this latter quantity estimated at the lens redshift. A direct fit, not taking into account the errors, gives:

$$
\begin{aligned}
& \log f_{\text {proj }}\left(R_{\mathrm{E}}\right)=-2.04+0.17 \log L_{V} \quad\left(\sigma_{\mathrm{rms}}=0.03\right), \\
& \log f_{\mathrm{DM}}\left(R_{\mathrm{E}}\right)=-2.66+0.22 \log L_{V} \quad\left(\sigma_{\mathrm{rms}}=0.04\right), \\
& \log f_{\mathrm{DM}}\left(R_{\mathrm{eff}}\right)=-2.41+0.20 \log L_{V} \quad\left(\sigma_{\mathrm{rms}}=0.04\right) .
\end{aligned}
$$

Note that the low scatter is actually far smaller than the typical uncertainty due to the errors on $\log L_{V}$ and $\Upsilon_{*}$, being of order 0.1 . The observed correlation with the luminosity suggests that a similar correlations should be observed with the total stellar mass. We indeed find:

$\log f_{\text {proj }}\left(R_{\mathrm{E}}\right)=-1.64+0.13 \log M_{\star}\left(\sigma_{\mathrm{rms}}=0.03\right)$,

$\log f_{\mathrm{DM}}\left(R_{\mathrm{E}}\right)=-1.98+0.15 \log M_{\star}\left(\sigma_{\mathrm{rms}}=0.05\right)$,

$\log f_{\mathrm{DM}}\left(R_{\mathrm{eff}}\right)=-1.74+0.15 \log M_{\star}\left(\sigma_{\mathrm{rms}}=0.05\right)$,

where $M_{\star}$ denotes here the total stellar mass. Although encouraging, such correlations should be taken with some caution because of the large measurement errors on the mass fractions and the relatively narrow luminosity and mass range probed (spanning roughly only one order of magnitude). It is therefore mandatory to both increase the sample and reduce the errors on the estimated stellar $M / L$ ratio before trying any detailed comparisons with available theoretical scenarios.
A different way of characterizing the galaxy DM content has been proposed by Napolitano et al. (2005) introducing the empirical DM gradient:

$\nabla_{l} \Upsilon=\frac{R_{\mathrm{eff}}}{r_{\text {out }}-r_{\text {in }}}\left[\left.\frac{M_{\mathrm{DM}}(r)}{M_{\star}(r)}\right|_{r=r_{\text {out }}}-\left.\frac{M_{\mathrm{DM}}(r)}{M_{\star}(r)}\right|_{r=r_{\text {in }}}\right]$

which, for our model, reduces to:

$\nabla_{l} \Upsilon=\frac{\left(\eta_{\text {out }}+\eta_{0}\right)^{\beta} \eta_{\text {out }}^{\alpha}-\left(\eta_{\text {in }}+\eta_{0}\right)^{\beta} \eta_{\text {in }}^{\alpha}}{\left[1-f_{\mathrm{DM}}\left(R_{\text {eff }}\right)\right]\left(\eta_{\text {out }}-\eta_{\text {in }}\right)\left(1+\eta_{0}\right)^{\beta}}$

We estimate this quantity for the lenses in our sample using the constraints from both the fit to the full lens sample and to the binned samples setting $\left(\eta_{\text {in }}, \eta_{\text {out }}\right)=(0.5,4.0)$ as in Napolitano et al. (2005). A comparison between the two is shown in the lower right panel in Fig. 2. Although the two set of constraints are in clear disagreement if we consider only the median values (with a direct fit giving $\nabla_{l} \Upsilon(B$ in $) \propto 0.83 \nabla_{l} \Upsilon(A l l)$ with a significant scatter), the error bars are so large that we can neglect this discrepancy and rely on the results of the fit to the full sample. The lower right panel in Fig. 3 then shows that this quantity is found to correlate with the luminosity. It is worth mentioning this trend with luminosity will be steeper if considering the fit binning the lenses, in agreement with a star formation efficiency decreasing with luminosity and mass (Napolitano et al. 2005). Using, however, the estimates from the fit to the full lens sample, we find:

$\log \nabla_{l} \Upsilon=-2.86+0.23 \log L_{V} \quad\left(\sigma_{\mathrm{rms}}=0.04\right)$

$\log \nabla_{l} \Upsilon=-2.53+0.20 \log M_{\star}\left(\sigma_{\mathrm{rms}}=0.04\right)$. 
Figure 6 of Napolitano et al. (2005) plots the estimated $\nabla_{l} \Upsilon$ values of a sample of local ETGs vs the total stellar mass, with $9.5 \leq \log M_{\star} \leq 12$. The empirical $M / L$ gradient seems to be a gently falling function of $\log M_{\star}$ over the range $9.5 \leq \log M_{\star} \leq$ 10.5 to become then an increasing function with a slope depending on the mass range considered. Indeed, over the mass range probed by our sample, the increase is quite gently (if not constant) so that our result $\nabla_{l} \Upsilon \propto M_{\star}^{0.20}$ is in qualitative good agreement with what may be inferred by the local sample. It is nevertheless worth stressing that our $\nabla_{l} \Upsilon$ values are consistent with those in Napolitano et al. (2005). Needless to say, a larger statistics is mandatory in oder to confirm these results and compare them with the trend predicted on the basis of dark halo models.

\subsection{The impact of the IMF choice}

All the results discussed insofar rely on the initial choice of the stellar IMF. Adopting a different IMF, indeed, changes the estimated stellar $M / L$ ratios $\Upsilon_{*}$ and hence the values of $M_{\text {vir }}$ and then of $\Upsilon_{\text {eff }}$ through Eq. (49). It is therefore worth wondering how the results change with the IMF. To this aim, we rescale all the stellar $M / L$ ratios by a factor 1.8 , that is the same as assuming a Salpeter rather than a Chabrier IMF. Then we repeat the fit to the full sample with these rescaled $\Upsilon_{*}$ and compare the best fit parameters and the marginalized constraints with those in Table 2.

Not surprisingly, we find a very good agreement for $(\alpha, \alpha+$ $\beta, s_{\mathrm{E}}$ ), while the only parameter changed is $\mu_{\mathrm{vir}}$. This can be easily understood considering that, for a given lens, rescaling $\Upsilon_{*}$ is the same as rescaling $\Upsilon_{\text {eff }}$ leaving unaltered the shape of the global $M / L$ ratio $\Upsilon(r)$. In order to still fit the data, we have therefore to retain the same functional expression and hence the same values of the slope parameters $\left(\alpha, \alpha+\beta, s_{\mathrm{E}}\right)$, but scale $\Upsilon_{\text {eff }}$ of the same amount as the stellar $M / L$ ratios. Since the luminosity is the same and the virial mass has to remain unchanged in order to give the same values of the projected mass and velocity dispersion, the virial DM mass fraction has to be rescaled according to:

$f_{\mathrm{vir}}^{S}=1-\Upsilon_{*}{ }^{S} L_{\mathrm{T}} / M_{\mathrm{vir}}=1-\left(1-f_{\mathrm{vir}}^{C}\right)\left(\Upsilon_{*}{ }^{S} / \Upsilon_{*}{ }^{C}\right)$

and for the mass scaling parameter we get:

$\frac{\mu_{\mathrm{vir}}^{S}}{\mu_{\mathrm{vir}}^{C}}=\left(\frac{M_{\mathrm{vir}}}{\Upsilon_{*}{ }^{S} L_{\mathrm{T}}}\right)\left(\frac{M_{\mathrm{vir}}}{\Upsilon_{*}{ }^{S} L_{\mathrm{T}}}\right)^{-1}=\frac{\Upsilon_{*}^{C}}{\Upsilon_{*}{ }^{S}} \simeq 0.55$

in agreement with what we find within the errors.

As a consequence, all the results obtained on the correlations between the DM mass fractions and the stellar luminosity and mass are still valid. Indeed, to take account of the change in the IMF, we have simply to rescale the $f_{\mathrm{DM}}$ values using $f_{\mathrm{DM}}^{S}=1-1.8\left(1-f_{\mathrm{DM}}^{C}\right)$, while $\log M_{\star}$ has to be increased by a constant factor $\simeq 0.25$. Both these changes obviously rescales the corresponding zeropoints and make the slope analyzed steeper than those obtained using a Chabrier IMF strongly pointing to an increasing DM content as a function of luminosity and stellar mass.

\section{Conclusions}

According to the estimated baryon and total matter density parameter predicted by the cosmological concordance model, DM should represent more than $80 \%$ of the total matter budget. As such, it is supposed to be ubiquitous representing therefore a significant component of any galactic structure. Notwithstanding this reasonable hypothesis, there is still a strong debate regarding the DM content of ETGs with a variety of dynamical, lensing and X-ray studies leading to contrasting results. The lack of a reliable mass tracer in the ETGs outer regions and modeling uncertainties make the problem of DM in ETGs a difficult one to conclusively address. In order to reduce any possible systematic bias due to the choice of the dark halo profile, a phenomenological approach has been proposed in Tortora et al. (2007) relying on the use of a versatile analytical expression for the global $M / L$ ratio $\Upsilon(r)$. Such a method allows to smoothly interpolate between the two extreme cases of light traces mass and dark halo dominance mimicking well the main dynamical properties of a large class of intermediate models. As a next mandatory step, we have here investigated the viability of the model by contrasting it against the measured Einstein angles and aperture velocity dispersions of a sample of 21 lenses observed by the SLACS survey. A Bayesian statistical analysis makes it possible to obtain the main results sketched below.

1. The effective galaxy model obtained by combining our ansatz for $\Upsilon(r)$ with the deprojection of the Sersic profile provided by the Prugniel \& Simien (1997) approximate expression is able to reproduce the observations under the assumptions that the slope parameters $(\alpha, \alpha+\beta)$, the logarithmic slope of the total density profile at the scaled Einstein radius, $s_{\mathrm{E}}=d \ln \rho / \mathrm{d} \ln \eta\left(\eta=R_{\mathrm{E}} / R_{\text {eff }}\right)$, and the virial mass ratio, $\mu_{\mathrm{vir}}=M_{\mathrm{vir}} / \Upsilon L_{\mathrm{T}}$, are universal quantities, while the model scalelelength $\eta_{0}=r_{0} / R_{\text {eff }}$ and global $M / L$ ratio at the effective radius $\Upsilon_{\text {eff }}$ may change on a case-by-case basis.

2. According to the marginalized constraints from the fit to the full lens sample, the slope parameters $\alpha$ and $\alpha+\beta$ are in agreement with what is expected in order the model mimics well cored halo profiles, while the total density profile is locally approximated by an isothermal model as yet found by previous studies in literature. We therefore argue that previous finding that an isothermal model fits the lensing and dynamics data is likely a consequence of having forced the total density profile to have a constant slope or of the data covering a too limited radial range to detect the actual change in the logarithimic slope.

3. Binning the lenses according to their absolute $V$ magnitude does not improve the fit and, on the contrary, gives constraints on both the model parameters and DM mass fractions that are in agreement (within 68 or $95 \%$ CL ranges) with those coming out from the fit to the full lens sample. However, the small statistics and the limited luminosity range probed does not allow us to draw any definitive conclusion on the universality of the proposed phenomenological $\Upsilon(r)$ model.

4. Using stellar population synthesis models and a Chabrier IMF to estimate the stellar $M / L$ ratios, we can constrain both the projected and spherical DM mass fractions at the Einstein and effective radii. Moreover, we have also estimated the empirical $M / L$ gradients proposed in Napolitano et al. (2005) as a different way to quantify the DM content in ETGs.

5. There is a clear trend of $f_{\mathrm{DM}}\left(R_{\text {eff }}\right)$ with both $\log L_{V}$ and $\log M_{\star}$ showing that the more luminous and/or massive a galaxy is, the larger is its DM content within the effective radius. Unfortunately, the ranges in $\log L_{V}$ and $\log M_{\star}$ probed are too small to make any quantitative comparison with theoretical models.

Although quite interesting and in good agreement with previous findings, the above results are still affected by too large uncertainties so that it is worth wondering how they can be 
strengthened. On one hand, one can simply increase the lens sample. Indeed, while this work was nearly at its end, the SLACS collaboration released a final catalog (Bolton et al. 2008) containing 170 lens candidates from which a subsample of 53 lenses with reliable determinations of the photometry, Einstein angle and aperture velocity dispersion. One can naively expect that more than doubling the number of objects will make our analysis more stringent making it possible to increase both the number of bins and the objects within a given bin. Unfortunately, more statistics does not automatically imply higher precision. This is likely the case for our model since the data are still probing only a limited range centred on $0.5-0.7 R_{\text {eff }}$ so that stacking more lenses does not increase the radial range probed. As a consequence, therefore, we do expect to narrow the constraints on the parameters mainly determing the shape of $\Upsilon(r)$ over this range (i.e., $\alpha$ and $s_{\mathrm{E}}$ ), while we cannot anticipate whether a similar improvement may be achieved for $\alpha+\beta$ and $\mu_{\mathrm{vir}}$ and hence $f_{\mathrm{DM}}\left(R_{\mathrm{eff}}\right)$. Therefore, rather than reducing the error on this quantity (that also depends on that on $\Upsilon_{*}$ and the photometric parameters $I_{\mathrm{e}}$ and $R_{\mathrm{eff}}$ ), we argue that such a worth to do analysis will improve the study of the $f_{\mathrm{DM}}\left(R_{\mathrm{eff}}\right)-L_{V}$ and $f_{\mathrm{DM}}\left(R_{\mathrm{eff}}\right)-M_{\star}$ relations thus improving the comparison with previous results and theoretical expectations. It is worth noting, however, that the SLACS survey selection function is biased against low mass systems (Bolton et al. 2006) so that such an analysis will likely be unable to explore the full ETGs mass range which could lessen the comparison with galaxy formation scenarios. Enlarging the sample will allow the possible physical interpretations of the trend we obtain to be better investigated. The change of DM within effective radius as a function of mass (luminosity) scale is the main driver of Fundamental Plane (Tortora et al. 2009b), due to the change of effective radius (Shankar \& Bernardi 2009) and/or star formation efficiency (Napolitano et al. 2005, 2009). Galaxy merging, close encounter, supernovae and AGN feedback could be the physical phenomena which give reason of these trends (Benson et al. 2000; Kaviraj et al. 2007; Khalatyan et al. 2008; Schawinski et al. Schawinski; Ruszkowski \& Springel 2009; Shankar \& Bernardi 2009; Tortora et al. 2009a).

As a complementary approach, one can resort to a different class of objects. Rather than concentrating on intermediate redshift galaxies, nearby ETGs may be investigated relying on the velocity dispersion profile to probe the model in the inner regions, while planetary nebulae may extend deep in the outer regions thus constraining the outer asymptotic slope. The analysis of the best fit residuals here obtained has shown that no correlation with the redshift is present thus suggesting that the model parameters $\left(\alpha, \alpha+\beta, s_{\mathrm{E}}, \mu_{\mathrm{vir}}\right)$ do not evolve. Such a conclusion must be, however, checked by both dividing lenses in redshift bins and looking at a nearby sample. Should this result be confirmed, one could then investigate what physical interplay between stellar and dark components is able to give rise to a global $M / L$ ratio whose shape does not evolve with $z$. In future analysis we will analyze this problem.

As a final comment, we would like to stress the virtue of a phenomenological approach (not necessarily our own) to the problem of DM in ETGs. A comparison with the determination of the dark energy equation of state $w(z)$ is worth doing here. Since a plethora of theoretically motivated models provide different expressions for $w(z)$, it has soon become clear that fitting all of them to the different data available would be a prohibitive task. This lead to the emergence of phenomenological proposals for $w(z)$ able to explore a wide range of models in a single step also providing model independent constraints to scrutinize different theories. Although the data on ETGs lensing and dynamics are still limited, it is nevertheless yet possible to rely on that positive experience to efficiently tackling the problem of DM in ETGs in a model independent way.

Acknowledgements. We warmly thank the referee for his/her constructive report which significantly helped us to improve the paper. V.F.C is supported by University of Torino and Regione Piemonte and partially from the INFN project PD51. C.T. is funded by a grant from the project Mecenas funded by the Compagnia di San Paolo.

\section{References}

Benson, A. J., Cole, S., Frenk, C. S., Baugh, C. M., \& Lacey, C. G. 2000, MNRAS, 311, 793

Binney, J. J., \& Evans, N. W. 2001, MNRAS, 327, L27

Bell, E. F., \& de Jong, R. S. 2001, ApJ, 550, 212

Bell, E. F., McIntosh, D. H., Katz, N., \& Weinberg, M. D. 2003, ApJS, 149, 289

Bolton, A. S., Burles, S., Koopmans, L. V. E., Treu, T., \& Moustakas, L. A. 2006, ApJ, 638, 703

Bolton, A. S., Burles, S., Koopmans, L. V. E., et al. 2008, [arXiv: 0805 . 1931] Borriello, A., \& Salucci, P. 2001, MNRAS, 323, 285

Borriello, A., \& Salucci, P., \& Danese, L. 2003, MNRAS, 341, 1109

Bryan, G. L., \& Norman, M. L. 1998, ApJ, 495, 80

Bruzual, G., \& Charlot, S. 2003, MNRAS, 344, 1000

Bullock, J. S., Kolatt, T. S., Sigad, Y., et al. 2001, MNRAS, 321, 559

Burkert, A. 1995, ApJ, 447, L25

Caon, N., Capaccioli, M., \& D’ Onofrio, M. 1993, MNRAS, 265, 1013

Cappellari, M., Bacon, R., Bureau, M., et al. 2006, MNRAS, 366, 1126

Cardone, V. F., Capozziello, S., Re, V., \& Piedipalumbo, E. 2001 A\&A, 379, 72

Cardone, V. F., Capozziello, S., Re, V., \& Piedipalumbo, E. 2002 A\&A, 382, 792

Cardone, V. F., Piedipalumbo, E., \& v Tortora, C. 2005, MNRAS, 358, 1325

Chabrier, G. 2001, ApJ, 554, 1274

Ciotti, L., \& Bertin, G. 1999, A\&A, 352, 477

Conroy, C., \& Wechsler, R. H. 2008, [arXiv:0805. 3346]

D' Agostini, G. 2004, [arXiv:physics/0403086]

de Vaucouleurs, G. 1948, Ann. Astroph., 11, 247

Dunkley, J., Bucher, M., Ferreira, P. G., Moodley, K., \& Skordis, C. 2005, MNRAS, 356, 925

Dunkley, J., Komatsu, E., Nolta, M. R., et al. 2009, ApJS, 180, 306

Einasto, J. 1965, Trudy Inst. Astrofiz. Alma - Ata, 5, 87

Einasto, J., \& Haud, U. 1989, A\&A, 223, 89

Fukushige, T., \& Makino, J. 2001, ApJ, 557, 533

Gavazzi, R., Treu, T., Rhodes, J. D., et al. 2007, ApJ, 667, 176

Gerhard, O., Kronawitter, A., Saglia, R. P., \& Bender, R. 2001, AJ, 121, 1936

Ghigna, S., Moore, B., Governato, F., et al. 2000, ApJ, 544, 616

Graham, A. W. 2002, MNRAS, 334, 859

Graham, A. W., \& Colless, M. 1997, MNRAS, 287, 221

Graham, A. W., \& Guzmán, R. 2003, AJ, 125, 2936

Graham, A. W., \& Driver, S. P. 2005, PASA, 22, 118

Guzik, J., \& Seljak, U. 2002, MNRAS, 335, 311

Jing, Y. P., \& Suto, Y. 2000, ApJ, 529, L69

Kaviraj S., Kirkby, L. A., Silk, J., \& Sarzi, M. 2007, MNRAS 382, 960

Keeton, C. R., Kochanek, C. S., \& Falco, E. E. 1998, ApJ, 509, 561

Khalatyan A., Cattaneo A., Schramm M., et al. 2008, MNRAS, 387, 13

Kochanek, C. S. 1995, ApJ, 445, 559

Koopmans, L. V. E., \& Treu, T. 2003a, ApJ, 583, 606

Koopmans, L. V. E., \& Treu, T. 2003b, ApJ, 599, 70

Koopmans, L. V. E., Treu, T., Bolton, A. S., Burles, S., \& Moustakas, L. A. 2006, ApJ, 649, 599

Kroupa P. 2001, MNRAS, 322, 231

Loewenstein, M., \& White, R. E. 1999 ApJ, 518, 50

Márquez, I., Lima Neto, G. B., Capelato, H., et al. 2001, A\&A, 379, 767

Mamon, G. A., \& Lokas, E. L. 2005a, MNRAS, 362, 95

Mamon, G. A., \& Lokas, E. L. 2005b, MNRAS, 363, 705

Mamon, G. A., Lokas, E. L., Dekel, A., Stoehr, F., \& Cox, T. J. 2006, [astro-ph/0601345]

Marinoni, C., \& Hudson, M. J., 2002, ApJ, 569, 101

Mazure, A., \& Capelato, H. V. 2002, A\&A, 383, 384

Merritt, D., Graham, A. W., Moore, B., Diemand, J., \& Terzic, B. 2006, AJ, 132, 2685

Moore, B., Governato, F., Qinn, T., Stadel, J., \& Lake, G. 1998, ApJ, 499, L5

Napolitano, N. R., Arnaboldi, M., Freeman, K. C., \& Capaccioli, M. 2001, A\&A, 377,784

Napolitano, N. R., Arnaboldi, M., \& Capaccioli, M., 2002, A\&A, 383, 791 
Napolitano, N. R., Capaccioli, M., Romanowsky, A. J., et al. 2005, MNRAS, 357,691

Napolitano, N. R., et al. 2009, MNRAS, in preparation

Navarro, J. F., Frenk, C. S., \& White, S. D. M. 1996, ApJ, 462, 563

Navarro, J. F., Frenk, C. S., \& White, S. D. M. 1997, ApJ, 490, 493

Navarro, J. F., Hayashi, E., Power, C., Jenkins, A. R., \& Frenck, C. S. 2004 MNRAS, 349, 1039

Padmanabhan, N., Seljak, U., Strauss, M. A., et al. 2004, New Ast., 9, 329

Persic, M., Salucci, P., \& Ashman, K. M. 1993, A\&A, 279, 343

Petters, A. O., Levine, H., \& Wambsganss, J. 2001, Singularity theory and gravitational lensing, Birkhäuser, Boston

Power, C., Navarro, J. F., Jenkins, A., et al. 2003, MNRAS, 338, 14

Prugniel, Ph., \& Simien, F. 1997, A\&A, 321, 111

Romanowsky, A. J., \& Kochanek, C. S. 1997, MNRAS, 287, 95

Romanowsky, A. J., \& Kochanek, C. S. 2001, ApJ, 553, 722

Romanowsky, A. J., Douglas, N. G., \& Arnaboldi, M. 2003, Science, 301, 1696

Ruszkowski M., \& Springel V. 2009, [arXiv: 0902 .0373]

Salpeter, E. E. 1955, 121, 161

Schawinski K., et al. 2008, [arXiv:0809.1096]
Schneider, P., Ehlers, J., \& Falco, E. E. 1992, Gravitational lenses (Berlin: Springer-Verlag)

Sersic, J. L. 1968, Atlas de Galaxies Australes, Observatorio Astronomico de Cordoba

Shankar, F., \& Bernardi, M. 2009, MNRAS, accepted, [arXiv:0903. 3964]

Spergel, D. N., et al. ApJS, 2003, 148, 175

Tortora, C., Cardone, V. F., \& Piedipalumbo, E. 2007, A\&A, 435, 107

Tortora C., Antonuccio-Delogu V., Kaviraj S., et al. 2008a, MNRAS in press, [arXiv:0903.0397]

Tortora, C., Napolitano, N. R., Romanowsky, A. J., et al. 2009, [arXiv:0901.3781]

Tortora, C., et al. 2009b, in preparation

Treu, T., \& Koopmans, L. V. E. 2002a, MNRAS, 337, L6

Treu, T., \& Koopmans, L. V. E. 2002b, ApJ, 575, 87

Treu, T., \& Koopmans, L. V. E. 2004, ApJ, 611, 739

van den Bosch, F. C., Yang, X., \& Mo, H. J. 2003a, MNRAS, 340, 771 van den Bosch, F. C., Mo, H. J., \& Yang, X. 2003b, MNRAS, 345, 923

van den Bosch, F. C., Yang, X., Mo, H. J., et al. 2007, MNRAS, 376, 841

Winn, J. N., Rusin, D., \& Kochanek, C. S. 2003, ApJ, 587, 80

Winn, J. N., Rusin, D., \& Kochanek, C. S. 2004, Nature, 427, 613 